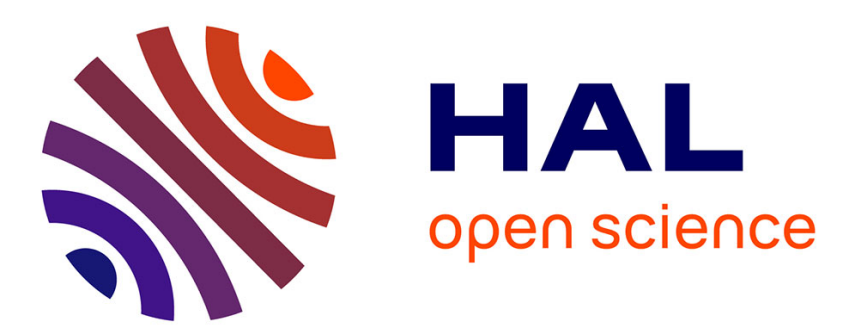

\title{
Switching rules for stabilization of linear systems of conservation laws
}

Pierre-Olivier Lamare, Antoine Girard, Christophe Prieur

\section{To cite this version:}

Pierre-Olivier Lamare, Antoine Girard, Christophe Prieur. Switching rules for stabilization of linear systems of conservation laws. SIAM Journal on Control and Optimization, 2015, 53 (3), pp.1599-1624. 10.1137/140953952 . hal-01165967

\section{HAL Id: hal-01165967 \\ https://hal.science/hal-01165967}

Submitted on 21 Jun 2015

HAL is a multi-disciplinary open access archive for the deposit and dissemination of scientific research documents, whether they are published or not. The documents may come from teaching and research institutions in France or abroad, or from public or private research centers.
L'archive ouverte pluridisciplinaire HAL, est destinée au dépôt et à la diffusion de documents scientifiques de niveau recherche, publiés ou non, émanant des établissements d'enseignement et de recherche français ou étrangers, des laboratoires publics ou privés. 


\title{
Switching rules for stabilization of linear systems of conservation laws *
}

\author{
Pierre-Olivier Lamare ${ }^{\dagger} \quad$ Antoine Girard ${ }^{\dagger} \quad$ Christophe Prieur ${ }^{\ddagger}$
}

\begin{abstract}
In this paper, the exponential convergence in $L^{2}$-norm is analyzed for a class of switched linear systems of conservation laws. The boundary conditions are subject to switches. We investigate the problem of synthesizing stabilizing switching controllers. By means of Lyapunov techniques, three control strategies are developed based on steepest descent selection, possibly combined with a hysteresis and a low-pass filter. For the first strategy we show the global exponential stabilizability, but no result for the existence and uniqueness of trajectories can be stated. For the other ones, the problem is shown to be well posed and global exponential convergence can be obtained. Moreover, we consider the robustness issues for these switching rules in presence of measurement noise. Some numerical examples illustrate our approach and show the merits of the proposed strategies. Particularly, we have developped a model for a network of open channels, with switching controllers in the gate operations.
\end{abstract}

\section{Introduction}

Physical networks may be represented by hyperbolic partial differential equations in one space dimension, yielding systems of conservation laws or balance laws. Among the different networks, we have in mind hydraulic networks (see [2], 6], 7]), road traffic networks (see [15]), or gas pipeline networks (see [10]). Due to the range of these applications in the engineering field, an important research on the theoretical aspects in modelling and control of such systems is in progress. The present paper deals with switched linear systems of conservation laws, and their control.

In such systems, some parameters may evolve in time. For example, it can be the boundary conditions in an open channel controlled by mobile spillways, or the state equations of a road where the speed limitation changes during the time. Of course, the state equations and the boundary conditions can both be subject to abrupt changes. In these cases, a switched system occurs: a system where there is both a continuous dynamics (given by partial differential equation) and a discrete dynamics (given by a switching rule). In the present work, only the boundary conditions are subject to switches.

Some results are available in literature for the stability and stabilizability of switched hyperbolic systems of balance laws or conservation laws. In [23] some sufficient conditions for the exponential stability are stated, uniformly with respect to a class of switching signals. In [1], a result of stability is given under an arbitrary switching signal using the propagation of the solution along the characteristics. In [11] a star-shaped network with a central node, and the wave equation governing on each edge, is studied. It is shown that a switching feedback stabilization at the exterior end of the edge is effective under some sufficient conditions. Moreover it is proved that the problem has solutions under the proposed switching rule. The well-posedness issue and the dependence of the solutions on the initial data for networked transport systems defined on directed

\footnotetext{
*This work has been partially supported by the LabEx PERSYVAL-Lab (ANR-11-LABX-0025) and by PHC TOURNESOL FR 2013.

${ }^{\dagger}$ Laboratoire Jean Kuntzmann, 51 rue des Mathématiques, Campus de Saint-Martin d'Hères, BP 53, 38041 Grenoble Cedex 09, France. Email:\{pierre-olivier.lamare, antoine.girard\}@imag.fr.

${ }^{\ddagger}$ Gipsa-lab, Department of Automatic Control, 11 Rue des Mathématiques, BP 46, 38402 Saint Martin d'Hères, France. Email: christophe.prieur@gipsa-lab.fr.
} 
graph is explored in [12, while an analysis based on functions of bounded variation in an extended way is used in [13 to prove well-posedness for a quasilinear system of first-order hyperbolic PDEs. In this last two references, the switching modeling is viewed as a tool for systems for which different time and spatial scales interact. It is also the approach used in [25, where a result of well-posedness is obtained for transport equation, in which the velocity term is subject to a closed-loop switching strategy.

An approach commonly used to study the stability and to design the boundary control of system of conservation laws is the Lyapunov analysis (see [4, 6]). In this paper we use this approach to design three swiching rules for our switched hyperbolic system of conservation laws. They are based on steepest descent selection, possibly combined with a hysteresis and a low-pass filter. We show that under a sufficient condition the global exponential convergence in $L^{2}$-norm is obtained. Moreover, we give a result of well-posedness of the problem for two of them. The sufficient condition takes the form of a matrix inequality. Hence the verification of this condition can be expressed as an optimization program, which can be solved numerically. Moreover an upper bound on the speed of convergence is given, and it is possible to numerically optimize it. Besides these results on stability we show that robustness with respect to measurement noise is obtained with mild modifications of the switching rules based on the hysteresis. The hysteresis properties were used for robustness as in 21, 22, 24, for non-linear finite-dimensional systems.

The paper is organized as follows. In Section 2, we introduce the class of switched linear hyperbolic systems of conservation laws considered in this paper and the question of existence of solutions in a general framework is discussed. In Section 3 , the common Lyapunov function used in our analysis is introduced, and some preliminary results are derived, then the three switching rules are proposed and analyzed. In Section 4 an abstract example is studied to illustrate our main results and show the effectiveness of switched systems, and finally a more realistic case is studied with a network of open-channels modelled with the so-called Saint-Venant equations.

This work is an extension of [17] where the results are stated without proofs and the well posedness is not investigated. Moreover the abstract example illustrates the new robustness results and the application to the control of a network of open channel has also been added in the present paper.

Notation. The set $\mathbb{R}^{+}$is the set of nonnegative real numbers. The set of square real matrices of dimension $n$ is denoted by $\mathbb{R}^{n \times n}$. Given a matrix $A$, the transpose of the matrix $A$ is denoted by $A^{\top}$. Given $n$ real numbers $a_{1}, \ldots, a_{n}$ the diagonal matrix $A \in \mathbb{R}^{n \times n}$ whose elements are these numbers is denoted by $\operatorname{diag}\left(a_{1}, \ldots, a_{n}\right)$. The identity matrix of dimension $n$ is denoted by $I_{n} .0$ denoted the zero matrix of suitable dimension according to the context. The entry $(i, j)$ of a matrix $A$ is denoted by $A[i, j]$. For a symmetric matrix $A \in \mathbb{R}^{n \times n}, A$ being positive definite is denoted $A>0$, while $A$ being positive semi-definite is denoted $A \geq 0$. The usual Euclidian norm in $\mathbb{R}^{n}$ is denoted by |.|, and the associated matricial norm in $\mathbb{R}^{n \times n}$ is denoted by $\|\cdot\|$. Let $I \subseteq \mathbb{R}$ and $J \subseteq \mathbb{R}^{p}$ for some $p \geq 1$. The set of functions $y: I \rightarrow J$ such that $|y|_{L^{p}(I, J)}^{p}=\int_{I}|y(x)|^{p} d x<\infty$, is denoted by $L^{p}(I, J)$. We denoted $y \in L_{\mathrm{loc}}^{p}(I, J)$ if $y \mathbb{1}_{K} \in L^{p}(I, J)$ for all compact subset $K$ of $I$, where $\mathbb{1}_{K}$ is the indicator function of $K$. The restriction of a function $y: I \rightarrow J$ on an open interval $\left(x_{1}, x_{2}\right) \subset I$ is denoted by $y_{\mid\left(x_{1}, x_{2}\right)}$.

\section{Switched Linear Hyperbolic System}

\subsection{Statement of the problem}

First let us define two function spaces with which we will deal in this paper.

Definition 1. Given an interval $I \subseteq \mathbb{R}$ and a set $J \subseteq \mathbb{R}^{n}$ for some $n \geq 1$, a piecewise left-continuous function (resp. a piecewise right-continuous function) $y: I \rightarrow J$ is a function continuous on each compact subset of $J$ except maybe on a finite number of points $x_{0}<x_{1}<\cdots<x_{p}$ such that for all $l \in\{0, \ldots, p-1\}$ there exists $y_{l}$ continuous on $\left[x_{l}, x_{l+1}\right]$ and $y_{l}=y_{\mid\left(x_{l}, x_{l+1}\right)}$. Moreover at the points $x_{1}, \ldots, x_{p}\left(\right.$ resp. $\left.x_{0}, \ldots, x_{p-1}\right)$ the function is continuous from the left (resp. from the right). The set of all piecewise left-continuous functions (resp. piecewise right-continuous functions) is denoted by $C_{l p w}(I, J)\left(\operatorname{resp} . C_{r p w}(I, J)\right.$ ).

Note that we have the following inclusions $C_{r p w}\left([0,1], \mathbb{R}^{n}\right), C_{l p w}\left([0,1], \mathbb{R}^{n}\right) \subset L^{2}\left([0,1], \mathbb{R}^{n}\right)$. 
Definition 2. Let $\mathcal{I}=\{1, \ldots, N\}$ be a finite set of modes. A switching signal is an element of $C_{r p w}\left(\mathbb{R}^{+}, \mathcal{I}\right)$. The times of discontinuities are called switching times.

The $n \times n$ switched linear hyperbolic system of conservation laws is then

$$
\begin{gathered}
\partial_{t} y(t, x)+\Lambda(t) \partial_{x} y(t, x)=0, \quad t \in \mathbb{R}^{+}, x \in[0,1], \\
y(t, 0)=G_{\sigma(t)} y(t, 1), \quad t \in \mathbb{R}^{+}, \\
y(0, x)=y^{0}(x), \quad x \in[0,1]
\end{gathered}
$$

where $y: \mathbb{R}^{+} \times[0,1] \rightarrow \mathbb{R}^{n}, \sigma: \mathbb{R}^{+} \rightarrow \mathcal{I}$ is the switching signal, and $y^{0} \in C_{l p w}\left([0,1], \mathbb{R}^{n}\right)$.

For all $i \in \mathcal{I}, G_{i}$ belongs to $\mathbb{R}^{n \times n}$, for all $t \in \mathbb{R}^{+}, \Lambda(t)$ is a diagonal positive definite matrix in $\mathbb{R}^{n \times n}$ i.e. $\Lambda(t)=\operatorname{diag}\left(\lambda_{1}(t), \ldots, \lambda_{n}(t)\right)$ where $\lambda_{1}(t), \ldots, \lambda_{n}(t)$ are in $L_{\text {loc }}^{1}\left(\mathbb{R}^{+}, \mathbb{R}^{+}\right)$and there exist $n$ pairs of real positive coefficients $\left(\underline{\lambda_{d}}, \overline{\lambda_{d}}\right), d=1, \ldots, n$ such that

$$
0<\underline{\lambda_{d}} \leq \lambda_{d}(t) \leq \overline{\lambda_{d}}, \quad \forall t \in \mathbb{R}^{+}, \forall d \in\{1, \ldots, n\} .
$$

In the sequel, we denote by $\underline{\Lambda}$ the diagonal matrix whose elements are the infinimum bound of the velocities $\Lambda(t)$ that is

$$
\underline{\Lambda}=\operatorname{diag}\left(\underline{\lambda_{1}}, \ldots, \underline{\lambda_{n}}\right)
$$

and we denote $\bar{\Lambda}$ the diagonal matrix whose elements are the supremum bound of the velocities $\Lambda(t)$ that is

$$
\bar{\Lambda}=\operatorname{diag}\left(\overline{\lambda_{1}}, \ldots, \overline{\lambda_{n}}\right) .
$$

We denote by $\underline{\lambda}$ and $\bar{\lambda}$ respectively the smallest eigenvalues of $\underline{\Lambda}$ and the largest eigenvalues of $\bar{\Lambda}$

$$
\begin{aligned}
& \underline{\lambda}=\min _{1 \leq i \leq n}\left\{\underline{\lambda_{i}}\right\}, \\
& \bar{\lambda}=\max _{1 \leq i \leq n}\left\{\overline{\lambda_{i}}\right\} .
\end{aligned}
$$

The aim of this work is to design a switching rule which depends only on the measurement at the boundary of the domain, in order to stabilize the system.

Indeed, hyperbolic systems, provided sensors are locally distributed, can be described with measurements at the boundaries. In our case, the system is only observed at the point $x=1$ at any time. The output is thus defined as

$$
w(t)=y(t, 1) .
$$

The output function is defined as soon as $y(t, \cdot) \in C_{l p w}\left([0,1], \mathbb{R}^{n}\right)$, since the evaluation of a function in $C_{l p w}\left([0,1], \mathbb{R}^{n}\right)$ is well-defined at every point in $[0,1]$.

The switching rule $\sigma$ is given as an output feedback law defined as follows

$$
\begin{array}{rlcc}
\sigma[w]: & \mathbb{R}^{+} & \rightarrow & \mathcal{I} \\
t & \mapsto & \sigma[w](t) .
\end{array}
$$

To summarize, the process evolves in a mode, a sensor measures the state $w(t)$ of the process at the boundary, then depending on this output, a switching rule imposes the mode in which the system must evolve afterwards. We make the following causality assumption on $\sigma$. For all $T \in \mathbb{R}^{+}$, for all $w, w^{\prime} \in C_{r p w}\left([0, T], \mathbb{R}^{n}\right)$, if

$$
w(t)=w^{\prime}(t), \quad \forall t \in[0, T]
$$

then we get

$$
\sigma[w](t)=\sigma\left[w^{\prime}\right](t), \quad \forall t \in[0, T] .
$$

Let us conclude this section with a last remark. 
Remark 1. In the system of equations (1), the matrix $\Lambda(t)$ is diagonal positive definite. This assumption is made only for the sake of simplicity in our analysis. Indeed we can consider more general diagonal matrices for $\Lambda(t)$. Suppose that there exists $m>0$ such that for all $t \in \mathbb{R}^{+}, \Lambda(t)$ is a diagonal matrix satisfying $\Lambda(t)=\operatorname{diag}\left(\lambda_{1}(t), \ldots, \lambda_{n}(t)\right)$ with $\lambda_{k} \in L_{\text {loc }}^{1}\left(\mathbb{R}^{+}, \mathbb{R}^{+}\right)$for $k \in\{1, \ldots, n\}$, and $0<\underline{\lambda^{+}} \leq \lambda_{k}(t) \leq \overline{\lambda^{+}}$for $k \in\{1, \ldots, m\}, \underline{\lambda^{-}} \leq \lambda_{k} \leq \overline{\lambda^{-}}<0$ for $k \in\{m+1, \ldots, n\}$. The matrix $\Lambda(t)$ is written as

$$
\Lambda(t)=\left(\begin{array}{cc}
\Lambda^{+}(t) & 0_{m, n-m} \\
0_{n-m, m} & \Lambda^{-}(t)
\end{array}\right),
$$

where $\Lambda^{+}(t)$ and $\Lambda^{-}(t)$ are respectively diagonal positive definite matrix and diagonal negative definite matrix. We introduce the notations $y^{+}=\left(y_{1}, \ldots, y_{m}\right)^{\top}, y^{-}=\left(y_{m+1}, \ldots, y_{n}\right)^{\top}$ such that $y=\left(y^{+}, y^{-}\right)^{\top}$. The system in its general form is

$$
\begin{gathered}
\partial_{t} y(t, x)+\Lambda(t) \partial_{x} y(t, x)=0, \quad t \in \mathbb{R}^{+}, x \in[0,1] \\
\left(\begin{array}{c}
y^{+}(t, 0) \\
y^{-}(t, 1)
\end{array}\right)=G_{\sigma(t)}\left(\begin{array}{c}
y^{+}(t, 1) \\
y^{-}(t, 0)
\end{array}\right), \quad t \in \mathbb{R}^{+}, \\
y(0, x)=y^{0}(x), \quad x \in[0,1]
\end{gathered}
$$

where for all $i \in \mathcal{I}, G_{i}=\left(\begin{array}{c}G_{i}^{++} G_{i}^{+-} \\ G_{i}^{-+}\end{array} G_{i}^{--}\right)$, such that $G_{i}^{++}, G_{i}^{--}, G_{i}^{+-}$and $G_{i}^{-+}$are matrices respectively in $\mathbb{R}^{m \times m}, \mathbb{R}^{(n-m) \times(n-m)}, \mathbb{R}^{m \times(n-m)}$ and $\mathbb{R}^{(n-m) \times m}$. The expression of $(9)$ is

$$
w(t)=\left(\begin{array}{l}
y^{+}(t, 1) \\
y^{-}(t, 0)
\end{array}\right) .
$$

By the change of variable $z(t, x)=\left(\begin{array}{c}y^{+}(t, x) \\ y^{-}(t, 1-x)\end{array}\right)$ we obtain a new system in the same form as $[1--22-(3)$.

\subsection{Solution of the system}

In this section we define the solution of the Cauchy problem associated with (1)-(2)-(3).

The solution will be defined with the classical method of characteristics (see 8 amongst other references). Let us recall the definition of characteristic curves.

Definition 3. Given $d$ in $\{1, \ldots, n\}$, the d-th characteristic is an absolutely continuous function $s \mapsto$ $X_{d}\left(s ; t^{\star}, x^{\star}\right)$ which satisfies $X_{d}\left(t^{\star} ; t^{\star}, x^{\star}\right)=x^{\star}$ and the ordinary differential equation

$$
\frac{d}{d s} X_{d}\left(s ; t^{\star}, x^{\star}\right)=\lambda_{d}(s)
$$

almost everywhere on the domain where $X_{d}\left(\cdot ; t^{\star}, x^{\star}\right)$ is defined.

Definition 4. Let $y^{0} \in C_{l p w}\left([0,1], \mathbb{R}^{n}\right)$ be given with $\sigma \in C_{r p w}\left(\mathbb{R}^{+}, \mathcal{I}\right)$. A solution to the Cauchy problem associated with (1)-(2)-(3) is a function $y: \mathbb{R}^{+} \times[0,1] \rightarrow \mathbb{R}^{n}$ such that, for every $\left(t^{\star}, x^{\star}\right) \in \mathbb{R}^{+} \times[0,1]$, the components of $y$ are satisfying the ordinary differential equations

$$
\frac{d}{d t} y_{d}\left(t, X_{d}\left(t ; t^{\star}, x^{\star}\right)\right)=0
$$

for every $t \geq t^{\star}$, such that $X_{d}\left(t ; t^{\star}, x^{\star}\right) \in[0,1]$, for all $d=1, \ldots, n$. Moreover the function $y$ satisfies the initial condition for $t=0$

$$
y_{d}(0, \cdot)=y_{d}^{0},
$$

together with the left boundary condition

$$
y_{d}(\cdot, 0)=\sum_{k=1}^{n} G_{\sigma(t)}[d, k] y_{k}(\cdot, 1),
$$

for all $d=1, \ldots, n$. 
Before stating any results on existence and uniqueness let us give some useful notations. Let $\kappa$ be the minimum time for a characteristic whose celerity is $\bar{\lambda}$ to cross the spatial domain $[0,1]$

$$
\kappa=\frac{1}{\bar{\lambda}}
$$

For $p \in \mathbb{N}$, let $\Delta_{p} \subset \mathbb{R}^{+}$be defined by

$$
\Delta_{p}=[p \kappa,(p+1) \kappa] .
$$

Proposition 1. Let $y^{0} \in C_{l p w}\left([0,1], \mathbb{R}^{n}\right)$, assume the strategy $\sigma$ is such that $\sigma[v] \in C_{r p w}\left(\mathbb{R}^{+}, \mathcal{I}\right)$ for all $v \in C_{r p w}\left(\mathbb{R}^{+}, \mathbb{R}^{n}\right)$. Then there exists a unique solution y to the closed loop switched system (1)-(2)-(3)-(9)10), and $w \in C_{\text {rpw }}\left(\mathbb{R}^{+}, \mathbb{R}^{n}\right)$.

Proof. We proceed by induction over the time interval $\Delta_{p}$. The first step consists to prove that $w \in$ $C_{r p w}\left(\Delta_{0}, \mathbb{R}^{n}\right)$.

For all $(t, x) \in \Delta_{0} \times[0,1]$ such that $\int_{0}^{t} \lambda_{d}(s) d s \leq x$ one gets, by the method of the characteristics,

$$
y_{d}(t, x)=y_{d}\left(0, x-\int_{0}^{t} \lambda_{d}(s) d s\right)=y_{d}^{0}\left(x-\int_{0}^{t} \lambda_{d}(s) d s\right), \quad d=1, \ldots, n .
$$

Let $t \in \Delta_{0}$. Since for all $d=1, \ldots, n$ and for all $s \in[0, t]$, we have $\lambda_{d}(s) \leq \bar{\lambda}$, one gets $\int_{0}^{t} \lambda_{d}(s) d s \leq 1$. Thus, the expression of $w$ on $\Delta_{0}$ is given by

$$
w_{d}(t)=y_{d}(t, 1)=y_{d}^{0}\left(1-\int_{0}^{t} \lambda_{d}(s) d s\right), \quad d=1, \ldots, n .
$$

Since $y^{0}$ is in $C_{l p w}\left([0,1], \mathbb{R}^{n}\right)$ it is clear that $w$ is in $C_{r p w}\left(\Delta_{0}, \mathbb{R}^{n}\right)$, hence by assumption and using the causality property $[11), 12, \sigma[w] \in C_{r p w}\left(\Delta_{0}, \mathcal{I}\right)$ is uniquely defined. The solution on $\Delta_{0}$ is given by

$$
y_{d}(t, x)=\left\{\begin{array}{l}
\sum_{k=1}^{n} G_{\sigma[w]\left(t-\tau_{d}(t, x)\right)}[d, k] w_{k}\left(t-\tau_{d}(t, x)\right), \quad \forall(t, x) \in \Delta_{0} \times[0,1] \text { such that } \int_{0}^{t} \lambda_{d}(s) d s>x, \\
y_{d}^{0}\left(x-\int_{0}^{t} \lambda_{d}(s) d s\right), \quad \forall(t, x) \in \Delta_{0} \times[0,1] \text { such that } \int_{0}^{t} \lambda_{d}(s) d s \leq x .
\end{array}\right.
$$

The function $\tau_{d}(t, x)$ is uniquely defined by the solution of the following equation

$$
\int_{t-\tau_{d}(t, x)}^{t} \lambda_{d}(s) d s=x
$$

Applying the implicit function theorem we can show that $\tau_{d}$ is continuous w.r.t. the time and space variables. Let $t \in \Delta_{0}$, since $w$ (resp. $\left.\sigma[w], y^{0}, \tau_{d}\right)$ belongs to $C_{r p w}\left(\Delta_{0}, \mathbb{R}^{n}\right)\left(\right.$ resp. to $C_{r p w}\left(\Delta_{0}, \mathcal{I}\right), C_{l p w}\left([0,1], \mathbb{R}^{n}\right)$, and $\left.C^{0}\left(\Delta_{0} \times[0,1], \mathbb{R}^{+}\right)\right)$, it follows from 24$)$ that $y(t, \cdot)$ is in $C_{l p w}\left([0,1], \mathbb{R}^{n}\right)$ for all $t \in \Delta_{0}$. This concludes the initial step of the induction.

Suppose for $p \geq 0, w \in C_{r p w}\left(\Delta_{p}, \mathbb{R}^{n}\right)$ and $y(t, \cdot) \in C_{l p w}\left([0,1], \mathbb{R}^{n}\right)$ for all $t \in \Delta_{p}$. Taking $y((p+1) \delta, \cdot)$ as the initial condition of the system and applying the same arguments as above we prove that $w \in$ $C_{r p w}\left(\Delta_{p+1}, \mathbb{R}^{n}\right)$ hence by assumption and using the causality property $[11),[12), \sigma[w] \in C_{r p w}\left(\Delta_{p+1}, \mathcal{I}\right)$ is uniquely defined, and $y$ exists on $\Delta_{p+1}$ with $y(t, \cdot)$ belongs to $C_{l p w}\left([0,1], \mathbb{R}^{n}\right)$ for all $t \in \Delta_{p+1}$, . Thus, we proved by induction that, for each $p \in \mathbb{N}, w \in C_{r p w}\left(\Delta_{p}, \mathbb{R}^{n}\right)$ and $y$ exists on $\Delta_{p}$ and $y(t, \cdot)$ belongs to $C_{l p w}\left([0,1], \mathbb{R}^{n}\right)$. Therefore, there exists a unique solution to the switched system (1)-(2)-(3)-(9)-(10), and $w \in C_{r p w}\left(\mathbb{R}^{+}, \mathbb{R}^{n}\right)$. It concludes the proof of Proposition 1 .

Remark 2. The solution $y$ of Proposition 1 , in general, does not depend continuously on the initial state $y^{0}$. This issue may be resolved by considering set-valued solution as in [12. The robustness of our control strategies will be discussed in Section 3 . 


\section{Stabilization}

In this paper we propose three control strategies. For the first one presented below, we are able to give only a result of global exponential stabilizability in $L^{2}$-norm and the existence of solution is not obtained in a general framework. For the next two strategies derived from the first one, we state existence, uniqueness of solution and global convergence in $L^{2}$-norm.

Let us start this section by a definition which will make clear what is meant by "convergence" for our switched system.

Definition 5. Given a switching rule $\sigma$, the closed loop system (1)-(2)-(3)-(9)-(10) is said to be globally exponentially convergent (in $L^{2}$-norm), if there exists a positive constant $\alpha$ and a function $g: \mathbb{R}^{+} \rightarrow \mathbb{R}^{+}$, such that for all $y^{0} \in C_{l p w}\left([0,1], \mathbb{R}^{n}\right)$, the solution of $(1)-(2)-(3)-(9)-(10)$ exists for all $t \in \mathbb{R}^{+}$and

$$
|y(t, \cdot)|_{L^{2}([0,1])} \leq e^{-\alpha t} g\left(\left|y^{0}\right|_{L^{2}([0,1])}\right),
$$

for all $t \in \mathbb{R}^{+}$. System (1)-(2)-(3)-(9)-(10) is said to be globally exponentially stabilizable (GES) if $g$ can be chosen linear.

With Definition 5 at hand, the next step is the analysis and the design of switching rules.

\subsection{Lyapunov function}

In this section, preliminary results on Lyapunov functions are derived. Following [4, the candidate Lyapunov function that is considered in this paper is written as, for all $y \in C_{l p w}\left([0,1], \mathbb{R}^{n}\right)$,

$$
V(y)=\int_{0}^{1} y(x)^{\top} Q y(x) e^{-\mu x} d x
$$

for a given diagonal positive definite matrix $Q \in \mathbb{R}^{n \times n}$ and a given $\mu>0$.

Let $y$ be a solution of system (1)-(2)-(3). We shall denote in the following

$$
\forall t \in \mathbb{R}^{+}, V=V(y(t, \cdot)) \text { and } \dot{V}=\frac{d}{d t} V(y(t, \cdot)) .
$$

With this notation we are able to state our first lemma, giving an inequality for the time derivative of $V$ along the solution of the switched system of conservation laws (1)-(2)-(3)-(9)-(10). This inequality will be useful to design the switching rules, and to give the proof of global exponential convergence of the system with them.

Lemma 1. The time derivative of the candidate Lyapunov function $V$ along a solution of (1)-(2)-(3)-(9)(10) satisfies

$$
\dot{V} \leq-2 \alpha V+q_{\sigma[w](t)}(w(t))
$$

where $q_{i}(w)=w^{\top}\left[G_{i}^{\top} Q \bar{\Lambda} G_{i}-e^{-\mu} Q \underline{\Lambda}\right] w$ and $\alpha=\frac{1}{2} \mu \underline{\lambda}$.

Proof. The time derivative of $V$ along the solutions of the switched system of conservation laws (1)-(2)-(3)- 
(9)- 10 is

$$
\begin{aligned}
\dot{V} & =2 \int_{0}^{1} y(t, x)^{\top} Q \partial_{t} y(t, x) e^{-\mu x} d x \\
& =-2 \int_{0}^{1} y(t, x)^{\top} Q \Lambda(t) \partial_{x} y(t, x) e^{-\mu x} d x \\
& =-\left[y(t, x)^{\top} Q \Lambda(t) y(t, x) e^{-\mu x}\right]_{x=0}^{x=1}-\mu \int_{0}^{1} y(t, x)^{\top} Q \Lambda(t) y(t, x) e^{-\mu x} d x \\
& =y(t, 0)^{\top} Q \Lambda(t) y(t, 0)-y(t, 1)^{\top} Q \Lambda(t) y(t, 1) e^{-\mu}-\mu \int_{0}^{1} y(t, x)^{\top} Q \Lambda(t) y(t, x) e^{-\mu x} d x \\
& =y(t, 1)^{\top}\left[G_{\sigma[w](t)}^{\top} Q \Lambda(t) G_{\sigma[w](t)}-Q \Lambda(t) e^{-\mu}\right] y(t, 1)-\mu \int_{0}^{1} y(t, x)^{\top} Q \Lambda(t) y(t, x) e^{-\mu x} d x .
\end{aligned}
$$

Since $Q$ is diagonal positive definite, using (7) it holds $Q \Lambda(t) \geq \underline{\lambda} Q$. Thus, using (5) and (6) we have

$\dot{V} \leq-2 \alpha \int_{0}^{1} y(t, x)^{\top} Q y(t, x) e^{-\mu x} d x+y(t, 1)^{\top}\left[G_{\sigma[w](t)}^{\top} Q \bar{\Lambda} G_{\sigma[w](t)}-Q \underline{\Lambda} e^{-\mu}\right] y(t, 1)=-2 \alpha V+q_{\sigma[w](t)}(w(t))$,

using the notation in (9). This concludes the proof of Lemma 1

Remark 3. Lemma 1 still holds when $Q$ is a symmetric, positive definite matrix such that $Q$ and $\Lambda(t)$ commute for all $t \in \mathbb{R}^{+}$.

\subsection{Switching strategies}

\subsubsection{Argmin}

In this section, we consider the closed-loop dynamics of the switched system of conservation laws (1)-(2)-(3) when using output controller (10). Following the idea developed in [9] and recalling the notation $q_{i}$ in Lemma 1. we define the memoryless switching rule

$$
\sigma[w](t)=\underset{i \in \mathcal{I}}{\arg \min } q_{i}(w(t)) .
$$

The idea of the argmin switching rule is to choose the mode which optimizes the decrease of the Lyapunov function at any time. So we need a condition which ensures that there always exists a mode for which the system is decreasing.

To study the convergence of the switched system of conservation laws (1)-(2)-(3)-(9)-(10) we need the following assumption.

Assumption 1. Let $\Gamma:=\left\{\gamma \in \mathbb{R}^{N} \mid \sum_{i=1}^{N} \gamma_{i}=1, \gamma_{i} \geq 0\right\}$. There exist $\gamma \in \Gamma$, a diagonal definite positive matrix $Q$ and a parameter $\mu>0$ such that

$$
\sum_{j=1}^{N} \gamma_{j}\left(G_{j}^{\top} Q \bar{\Lambda} G_{j}-e^{-\mu} Q \underline{\Lambda}\right) \leq 0 .
$$

Assumption 1 implies that there always exits a mode $i \in \mathcal{I}$ such that $q_{i}(w(t)) \leq 0$. Thus we can give our first result of global exponential stabilizability of the system (11)-(2)-(3)-(9)-(10) with the argmin switching rule. 
Remark 4. An important issue is the numerical computation of $\gamma \in \Gamma, \mu>0$ and of a diagonal positive definite matrix $Q$ such that Assumption 1 holds. The problem is bilinear in $Q, \gamma$ and $e^{-\mu}$ and the numerical verification of Assumption 1 can be quite complex especially for larger $N$, since we need to solve a Bilinear Matrix Inequalities (BMI) in $\gamma, e^{-\mu}$ and $Q$. A special case is when $N=2$, a solution consists in performing a line search over the parameters $\gamma$ and $\mu$ and, for each pair $(\gamma, \mu)$, to solve a convex problem in the variables $Q$ written in terms of the Linear Matrix Inequality (LMI) (31). This can be done numerically in polynomial time. It is the approach taken for the two examples given in Section 4. See 27] for a tutorial on LMI and BMI problems.

Proposition 2. Under Assumption (1, system (1)-(2)-(3)-(9)-(10) with switching rule (30) is such that, as long as the solution exists,

$$
|y(t, \cdot)|_{L^{2}([0,1])} \leq c e^{-\alpha t}\left|y^{0}\right|_{L^{2}([0,1])},
$$

where $\alpha=\frac{1}{2} \mu \underline{\lambda}$, and $c>0$.

Proof. Consider the candidate Lyapunov function (27). Thanks to Lemma 1 and using the switching rule (30) we have, along the solution $y$,

$$
\dot{V} \leq-2 \alpha V+q_{\sigma[w](t)}(w(t))=-2 \alpha V+\min _{i \in \mathcal{I}} q_{i}(w(t)) .
$$

By Assumption 1 there exists $\gamma \in \Gamma$ such that $\sum_{j=1}^{N} \gamma_{j}\left(G_{j}^{\top} Q \bar{\Lambda} G_{j}-e^{-\mu} Q \underline{\Lambda}\right) \leq 0$. Therefore, $\forall t \in \mathbb{R}^{+}$, $\sum_{j=1}^{N} \gamma_{j} q_{j}(w(t)) \leq 0$. Hence $\forall t \in \mathbb{R}^{+}$there exists $i \in \mathcal{I}$ such that $q_{i}(w(t)) \leq 0$, which gives $\dot{V} \leq-2 \alpha V$. Hence $V$ satisfies $V \leq e^{-2 \alpha t} V\left(y^{0}\right)$. Moreover there exists $\kappa>0$ (e.g. $\kappa$ could be the largest eigenvalue of $Q$ ) such that $Q \leq \kappa I_{n}$. Thus the inequality $V \leq \kappa e^{-2 \alpha t}\left|y^{0}\right|_{L^{2}([0,1])}^{2}$, holds. In the same manner, there exists $\nu>0$ (e.g. $\nu$ could be the smallest eigenvalue of $Q$ ) such that $\nu I_{n} \leq Q$. Since there exists a $\delta>0$ such that $\delta \leq e^{-\mu x} \leq 1, \forall x \in[0,1]$, the inequality $\nu \delta|y|_{L^{2}([0,1])}^{2} \leq V$ holds. Finally, the inequality $|y|_{L^{2}([0,1])} \leq \sqrt{\frac{\kappa}{\nu \delta}} e^{-\alpha t}\left|y^{0}\right|_{L^{2}([0,1])}$ holds. Therefore the switched hyperbolic system [1)- $22-(3 p-(9)-(10)$ is globally exponentially stabilizable (taking $g(r)=\sqrt{\frac{\kappa}{\nu \delta}} r$ ) with the argmin startegy. This conludes the proof of Proposition 2 .

Remark 5. The major drawback of this switching rule is the possibility of a finite time of existence for the solution, as illustrated by the following example. Let us consider the two transport equations

$$
\partial_{t}\left(\begin{array}{l}
y_{1} \\
y_{2}
\end{array}\right)+\left(\begin{array}{ll}
1 & 0 \\
0 & 1
\end{array}\right) \partial_{x}\left(\begin{array}{l}
y_{1} \\
y_{2}
\end{array}\right)=0
$$

with the boundary matrices

$$
G_{1}=\left(\begin{array}{cc}
0.99 & -0.99 \\
0 & 0
\end{array}\right), \quad G_{2}=\left(\begin{array}{cc}
0 & 0 \\
-0.99 & -0.99
\end{array}\right)
$$

The initial condition is chosen as

$$
y^{0}(x)=\left(\begin{array}{c}
f(x) \\
x
\end{array}\right), \quad x \in[0,1]
$$

where

$$
f(x)= \begin{cases}0 & \text { if } x=0 \\ x \sin \left(\frac{1}{x}\right) & \text { if } x \neq 0\end{cases}
$$

Assumption 1 is checked with $Q=I_{2}, \mu=10^{-3}$ and $\gamma_{1}=\gamma_{2}=0.5$

$$
\sum_{i=1}^{2} 0.5\left(G_{i}^{\top} Q \Lambda G_{i}-e^{-\mu} Q \Lambda\right)=\left(\begin{array}{cc}
-0.0189 & 0 \\
0 & -0.0189
\end{array}\right)
$$


On Figure 1 the conic regions corresponding to $q_{i}(w)<0, i=1,2$ are depicted, giving for the switching rule (30),

$$
\sigma[w](t)= \begin{cases}1 & \text { if } w_{1} w_{2}>0 \\ 2 & \text { if } w_{1} w_{2}<0\end{cases}
$$

Moreover the system trajectory for $t \in[0,1)$ is depicted on Figure 1

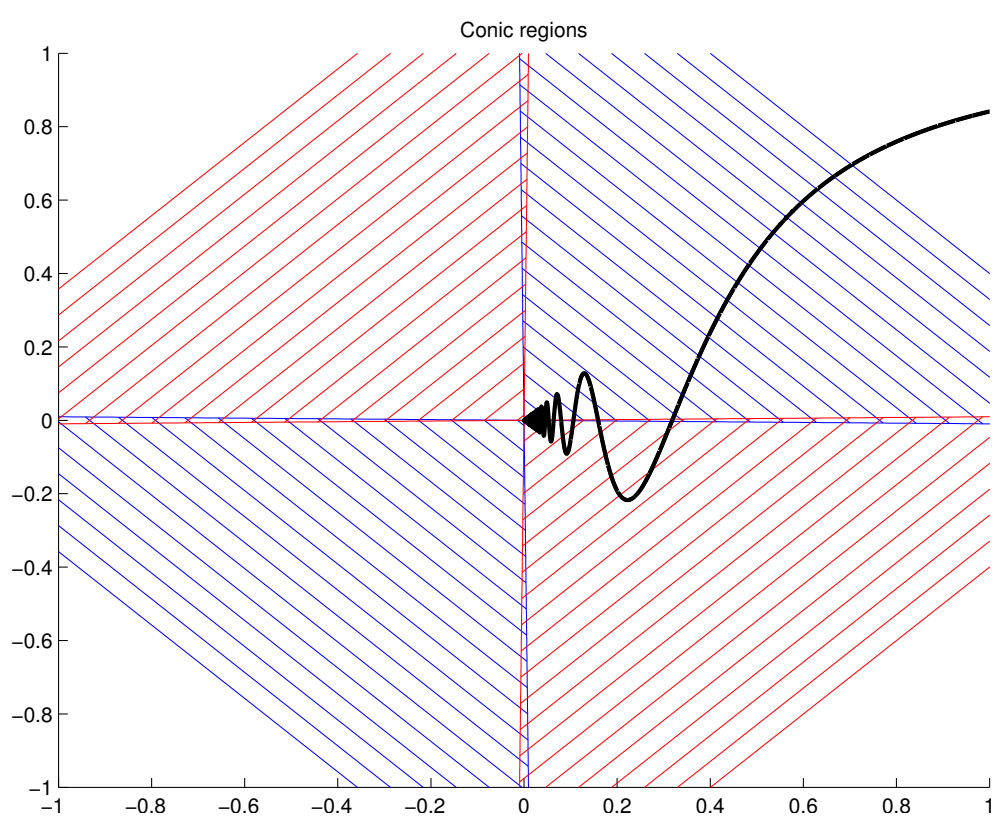

Figure 1: Conic regions where each individual system is active, and the trajectory of $w(t)$ for $t \in[0,1)$

One can see that when the time approaches 1 , with the switching rule 30 the solution cross an infinite number of times the thin overlap region, leading to the Zeno behavior (see [18]), that is an accumulation of switching event at a particular instant. Thus the solution is not defined for all positive time but only on $[0,1)$.

\subsubsection{Argmin with hysteresis}

The first result shows that under Assumption 1 the switched system of conservation laws (1)-(2)-(3)-(9)-(10) with the argmin switching rule is globally exponentially stabilizable. The limitation of this rule is a possible fast switching behavior (see [17] and the example therein for an illustration of such behavior), and from a theoretical point of view leading to the absence of solution beyond a given time. From a pratical point of view this fast switching is undesirable. So the goal is to use strategies to slow down the switching. The first one is the hysteresis strategy.

First, we will show that under this switching rule and Assumption 1 the system is well-posed.

For all $t>0$ we denote by $\sigma[w]\left(t^{-}\right)$the limit from the left of $t$ of the value of $\sigma[w](t)$. Roughly speaking, it is the value of $\sigma[w]$ "just before $t$ ".

The strategy is the following

$$
\begin{aligned}
\sigma[w](t) & = \begin{cases}\sigma[w]\left(t^{-}\right) & \text {if } q_{\sigma[w]\left(t^{-}\right)}(w(t))<\varepsilon(t), \\
\underset{j \in\{1, \ldots, N\}}{\arg \min } q_{j}(w(t)) & \text { if } q_{\sigma[w]\left(t^{-}\right)}(w(t)) \geq \varepsilon(t),\end{cases} \\
\sigma[w](0) & =\underset{j \in\{1, \ldots, N\}}{\arg \min } q_{j}(w(0)), \\
\dot{\varepsilon}(t) & =-\eta \varepsilon(t), \quad \varepsilon(0)>0,
\end{aligned}
$$


where $\eta$ is a positive coefficient such that $\eta>2 \alpha$.

Lemma 2. Under Assumption 1 with the strategy defined in $(38)-(40)$, if $w \in C_{r p w}\left(\mathbb{R}^{+}, \mathbb{R}^{n}\right)$ then $\sigma[w] \in$ $C_{r p w}\left(\mathbb{R}^{+}, \mathcal{I}\right)$.

Proof. Let $K$ be a compact subset of $\mathbb{R}^{+}$. Let us show that the number of discontinuities of $\sigma[w]$ is finite in $K$. By hypothesis $w$ has a finite number of discontinuities on $K$. Let $t_{1}, \ldots, t_{M} \in K$ be these times of discontinuity, and $t_{0}$ and $t_{M+1}$ are respectively the lower bound and the upper bound of the interval $K$.

Pick $i \in\{0, \ldots, M\}$. We need to estimate the number of discontinuities of $\sigma[w]$ on each time interval $\left[t_{i}, t_{i+1}\right]$. We can define $\tilde{w}$ as the continuation of $w$ to the time interval $\left[t_{i}, t_{i+1}\right]$ with the left limit of $w$ in $t_{i+1}$, that is

$$
\begin{aligned}
\tilde{w}(t) & =w(t), \quad \text { if } t \in\left[t_{i}, t_{i+1}\right), \\
\tilde{w}\left(t_{i+1}\right) & =\lim _{t \rightarrow t_{i+1}^{-}} w(t) .
\end{aligned}
$$

The definition of $C_{r p w}\left(\mathbb{R}^{+}, \mathbb{R}^{n}\right)$ insures that the left limit of $w$ exists and that $\tilde{w}$ is continuous on $\left[t_{i}, t_{i+1}\right]$. The number of discontinuities of $\sigma[w]$ on the time interval $\left[t_{i}, t_{i+1}\right)$ is less than or possibly equal to the number of discontinuities when considering $\sigma[\tilde{w}]$ on $\left[t_{i}, t_{i+1}\right]$.

Now observe that on $\mathbb{R}^{n}, q_{k}$ is continuous, as $\tilde{w}$ on $\left[t_{i}, t_{i+1}\right]$, thus the functions $q_{k}(\tilde{w})$ are continuous on the compact $\left[t_{i}, t_{i+1}\right]$ and therefore uniformly continuous. Using the fact that there is a finite number of functions $q_{k}(\tilde{w})$ and the uniform continuity, there exists $\tau_{i}^{*}>0$ such that

$$
\forall k \in \mathcal{I}, \quad \forall \hat{t}_{i}, \check{t}_{i} \in\left[t_{i}, t_{i+1}\right]: \quad\left|\hat{t}_{i}-\check{t}_{i}\right| \leq \tau_{i}^{*} \Rightarrow\left|q_{k}\left(\tilde{w}\left(\hat{t}_{i}\right)\right)-q_{k}\left(\tilde{w}\left(\check{t}_{i}\right)\right)\right| \leq \varepsilon\left(t_{i+1}\right) .
$$

Due to Assumption 1 at a switching time $t, q_{\sigma[\tilde{w}](t)}(\tilde{w}(t)) \leq 0$, and therefore the parameter $\tau_{i}^{*}$ gives a lower bound for the distance between two switches. Thus an upper bound for the maximal number of switches on $\left[t_{i}, t_{i+1}\right]$ is given by

$$
\bar{s}_{i}=\frac{t_{i+1}-t_{i}}{\tau_{i}^{*}}+1
$$

To conclude we get that the number of discontinuities of $\sigma[w]$ on $K$ is bounded by

$$
\bar{S}=\sum_{i=1}^{M} \bar{s}_{i}
$$

which is finite. Note that the right continuity of $\sigma[w]$ follows from the strict inequality in the first line of (38). It concludes the proof of Lemma 2 .

With the above lemma, the following theorem can be stated.

Theorem 1. Under Assumption 1 , system (1)-(2)-(3)-(9)-(10) with an initial condition $y^{0} \in C_{l p w}\left([0,1], \mathbb{R}^{n}\right)$ and the switching rule $(38)-(40)$ is globally exponentially convergent with $\alpha=\frac{1}{2} \mu \underline{\lambda}$ and $g(r)=c(r+\sqrt{\varepsilon(0)})$ with $c>0$.

Proof. The existence and uniqueness of a solution to the system (1)-(2)-(3)-(9)-(10) with the switching rule (38) - 40 is given by Lemma 2 and Proposition 1. Now to show that the system is globally exponentially convergent we have to etablish relation (26). Thanks to Lemma 1 the time derivative of $V$ along the solution of $(1)-(2)-(3)-(9)-(10)$ is

$$
\dot{V} \leq-2 \alpha V+q_{\sigma[w](t)}(w(t)) .
$$

Since the invariant in the argmin with hysteresis switching rule is that $q_{\sigma[w](t)}(w(t)) \leq \varepsilon(t)$ at any time $t \in \mathbb{R}^{+}$, it gives

$$
\dot{V} \leq-2 \alpha V+\varepsilon(t)
$$


Then using the Gronwall's Lemma, one gets

$$
V \leq e^{-2 \alpha t} V\left(y^{0}\right)+e^{-2 \alpha t} \int_{0}^{t} e^{2 \alpha s} \varepsilon(s) d s .
$$

Using the expression of $\varepsilon$ it follows

$$
V \leq e^{-2 \alpha t} V\left(y^{0}\right)+\frac{\varepsilon(0)}{2 \alpha-\eta} e^{-\eta t}-\frac{\varepsilon(0)}{2 \alpha-\eta} e^{-2 \alpha t} .
$$

Letting $c=\max \left\{\sqrt{\frac{\kappa}{\nu \delta}}, \frac{1}{\sqrt{\nu \delta(\eta-2 \alpha)}}\right\}$ one gets 26 with $g(r)=c(r+\sqrt{\varepsilon(0)})$, and thus the exponential convergence of (1)-(2)-(3)-(9)-(10) with the switching rule (38)-(40) follows. This concludes the proof of Theorem 1

Remark 6. Let us note that the function $\varepsilon(t)>0$ is essential to have the existence of solution for all time. Indeed, looking at the example given in Remark 5 , one can see that the argmin with hysteresis strategy for which $\varepsilon(t)=0$ leads to the same problem as for the argmin strategy alone.

\section{Robustness with respect to measurement noise}

The switching rule (38)- 40 depends on the measurements at the boundary. A natural question which arises is how the system reacts in presence of measurement noise. In order to establish a result of stability in presence of measurement noise, let us consider system (1)-(2)-(3) with

$$
\sigma(t)=\sigma[w+\delta](t),
$$

where $w$ is given by $(9)$ as before and $\delta \in C_{r p w}\left(\mathbb{R}^{+}, \mathbb{R}^{n}\right)$ is an unknown disturbance. The following assumption is stated.

Assumption 2. Let $\Gamma:=\left\{\gamma \in \mathbb{R}^{N} \mid \sum_{i=1}^{N} \gamma_{i}=1, \gamma_{i} \geq 0\right\}$. There exist $\gamma \in \Gamma$, a diagonal definite positive matrix $Q$ and a parameter $\mu>0$ such that

$$
\sum_{j=1}^{N} \gamma_{j}\left(G_{j}^{\top} Q \bar{\Lambda} G_{j}-e^{-\mu} Q \underline{\Lambda}\right)<0 .
$$

It implies that there exists $\zeta>0$ such that

$$
\sum_{j=1}^{N} \gamma_{j}\left(G_{j}^{\top} Q \bar{\Lambda} G_{j}-e^{-\mu} Q \underline{\Lambda}\right) \leq-\zeta I_{n} .
$$

This former Assumption is stronger than Assumption 1 by comparing (31) with 49). Let us consider the switching rule adapted from (38)-40.

$$
\begin{aligned}
\sigma[w+\delta](t) & = \begin{cases}\sigma[w+\delta]\left(t^{-}\right) & \text {if } q_{\sigma[w+\delta]\left(t^{-}\right)}(w(t)+\delta(t))<-\zeta|w(t)+\delta(t)|^{2}+\varepsilon(t) \\
\underset{j \in\{1, \ldots, N\}}{\arg \min } q_{j}(w(t)+\delta(t)) & \text { if } q_{\sigma[w+\delta]\left(t^{-}\right)}(w(t)+\delta(t)) \geq-\zeta|w(t)+\delta(t)|^{2}+\varepsilon(t)\end{cases} \\
\sigma[w+\delta](0) & =\underset{j \in\{1, \ldots, N\}}{\arg \min } q_{j}(w(0)+\delta(0)), \\
\dot{\varepsilon}(t) & =-\eta \varepsilon(t), \quad \varepsilon(0)>0,
\end{aligned}
$$

where $\zeta$ and $\eta$ are positive constants and $\eta>2 \alpha$. Let us define the parameter

$$
\beta=\sup _{t} \max _{i \in \mathcal{I}}\left\|G_{i}^{\top} Q \Lambda(t) G_{i}-e^{-\mu} Q \Lambda(t)+\zeta I_{n}\right\|,
$$


Remark 7. Note that $\beta$ is the supremum of a time-dependent matrix norm. Nonetheless, this value is finite due to the bounds (4) on the matrix $\Lambda$. For instance, one has

$$
\beta \leq \max _{i \in \mathcal{I}}\|Q\|\|\bar{\Lambda}\|\left\|G_{i}\right\|^{2}+e^{-\mu}\|Q\|\|\bar{\Lambda}\|+\zeta .
$$

Let us state the following result.

Proposition 3. Under Assumption 2, system (1)-(2)-(3)-(99)-(47) with an initial condition $y^{0} \in C_{l p w}\left([0,1], \mathbb{R}^{n}\right)$ and a disturbance $\delta \in C_{r p w}\left(\mathbb{R}^{+}, \mathbb{R}^{n}\right)$ satisfying

$$
|\delta(t)| \leq \rho|w(t)|, \quad \forall t \in \mathbb{R}^{+},
$$

for a positive constant $\rho$ such that

$$
0<\rho \leq \sqrt{1+\frac{\zeta}{\beta}}-1,
$$

with the switching rule 50-52) is globally exponentially convergent.

Proof. First note that Lemma 2 is still valid in the context of the switching rule $(50)-(52)$ instead of $\sqrt{38})-(40)$, for any $\delta \in C_{r p w}\left(\mathbb{R}^{+}, \mathbb{R}^{n}\right)$. Hence the existence and uniqueness of a solution to the system (1)-(2)-(3)-(9)(47) with the switching rule (50)-(52) is given by Lemma 2 and Proposition 1. Due to Lemma 1 the time derivative of $V$ is given by

$$
\dot{V} \leq-2 \alpha V+q_{\sigma[w+\delta](t)}(w(t)) .
$$

The quadratic term in the right-hand part of (57) can be written as

$$
\begin{aligned}
q_{\sigma[w+\delta](t)}(w(t))= & q_{\sigma[w+\delta](t)}(w(t)+\delta(t)) \\
& -\delta(t)^{\top}\left(G_{\sigma[w+\delta](t)}^{\top} Q \Lambda(t) G_{\sigma[w+\delta](t)}-e^{-\mu} Q \Lambda(t)\right)(2 w(t)+\delta(t)) .
\end{aligned}
$$

By Assumption 2 and 49, ,50 one gets

$$
\begin{aligned}
q_{\sigma[w+\delta](t)}(w(t)) \leq & -\zeta|w(t)+\delta(t)|^{2}+\varepsilon(t) \\
& -\delta(t)^{\top}\left(G_{\sigma[w+\delta](t)}^{\top} Q \Lambda(t) G_{\sigma[w+\delta](t)}-e^{-\mu} Q \Lambda(t)\right)(2 w(t)+\delta(t)) .
\end{aligned}
$$

Expanding $-\zeta|w(t)+\delta(t)|^{2}$ one has

$$
\begin{aligned}
q_{\sigma[w+\delta](t)}(w(t)) \leq & -\zeta|w(t)|^{2}+\varepsilon(t)-\zeta|\delta(t)|^{2}-2 \zeta \delta(t)^{\top} w(t) \\
& -\delta(t)^{\top}\left(G_{\sigma[w+\delta](t)}^{\top} Q \Lambda(t) G_{\sigma[w+\delta](t)}-e^{-\mu} Q \Lambda(t)\right)(2 w(t)+\delta(t)) .
\end{aligned}
$$

Rearranging the three last terms of 600 as follows

$$
\begin{aligned}
& -\zeta|\delta(t)|^{2}-2 \zeta \delta(t)^{\top} w(t)-\delta(t)^{\top}\left(G_{\sigma[w+\delta](t)}^{\top} Q \Lambda(t) G_{\sigma[w+\delta](t)}-e^{-\mu} Q \Lambda(t)\right)(2 w(t)+\delta(t)) \\
= & -\delta(t)^{\top}\left(G_{\sigma[w+\delta](t)}^{\top} Q \Lambda(t) G_{\sigma[w+\delta](t)}-e^{-\mu} Q \Lambda(t)+\zeta I_{n}\right) 2 w(t) \\
& -\delta(t)^{\top}\left(G_{\sigma[w+\delta](t)}^{\top} Q \Lambda(t) G_{\sigma[w+\delta](t)}-e^{-\mu} Q \Lambda(t)+\zeta I_{n}\right) \delta(t) \\
= & -\delta(t)^{\top}\left(G_{\sigma[w+\delta](t)}^{\top} Q \Lambda(t) G_{\sigma[w+\delta](t)}-e^{-\mu} Q \Lambda(t)+\zeta I_{n}\right)(2 w(t)+\delta(t)),
\end{aligned}
$$

we get

$$
\begin{aligned}
q_{\sigma[w+\delta](t)}(w(t)) \leq & -\zeta|w(t)|^{2}+\varepsilon(t) \\
& -\delta(t)^{\top}\left(G_{\sigma[w+\delta](t)}^{\top} Q \Lambda(t) G_{\sigma[w+\delta](t)}-e^{-\mu} Q \Lambda(t)+\zeta I_{n}\right)(2 w(t)+\delta(t)) .
\end{aligned}
$$


Using (62), (53) and (55) we get

$$
q_{\sigma[w+\delta](t)}(w(t)) \leq\left(\beta \rho^{2}+2 \beta \rho-\zeta\right)|w(t)|^{2}+\varepsilon(t) .
$$

In order to have $q_{\sigma[w+\delta](t)}(w(t)) \leq \varepsilon(t)$ we need $\beta \rho^{2}+2 \beta \rho-\zeta \leq 0$. This expression is a polynomial in $\rho$. Computing the discriminant one gets

$$
\Delta=4 \beta^{2}+4 \beta \zeta>0 .
$$

Then there exists two real roots for this polynomial. Since the constant term is negative, the two roots have opposite sign. Hence there exists $\rho$ such that

$$
0<\rho \leq \sqrt{1+\frac{\zeta}{\beta}}-1,
$$

for which one has $q_{\sigma[w+\delta](t)}(w(t)) \leq \varepsilon(t)$. The end of the proof follows the proof of Theorem 1 . This concludes the proof of Proposition 3

\subsubsection{Argmin, hysteresis and filter}

Thanks to Lemma 1 it holds along the solutions of (1)-(2)-(3)-(9)-(10)

$$
\dot{V} \leq-2 \alpha V+q_{\sigma[w](t)}(w(t)) .
$$

Keeping in mind the objective of decreasing the number of switches, a low-pass filter is added to the switching rule (38)- 40 : instead of imposing that $q_{\sigma[w](t)}(w(t)) \leq \varepsilon(t)$ at any time $t \geq 0$, we just impose that a weighted averaged value of $q_{\sigma[w](s)}(w(s))$ is less than $\varepsilon(t)$.

Let us define a function $m \in C_{r p w}\left(\mathbb{R}^{+}, \mathbb{R}\right)$ and a switching rule $\sigma[w]$ such that

$$
\begin{aligned}
& m(0)=0 \text {, } \\
& \lim _{\tau \rightarrow t^{+}} m(\tau)= \begin{cases}m(t) & \text { if } m(t)<\varepsilon(t), \\
0 & m(t)=\varepsilon(t),\end{cases} \\
& \sigma[w](t)= \begin{cases}\sigma[w]\left(t^{-}\right) & \text {if } m(t)<\varepsilon(t), \\
\underset{j \in\{1, \ldots, N\}}{\arg \min } q_{j}(w(t)) & \text { if } m(t)=\varepsilon(t),\end{cases} \\
& \sigma[w](0)=\underset{j \in\{1, \ldots, N\}}{\arg \min } q_{j}(w(0)), \\
& \dot{\varepsilon}(t)=-\eta \varepsilon(t), \quad \varepsilon(0)>0,
\end{aligned}
$$

where $\eta$ is such that $\eta>2 \alpha, \alpha=\frac{1}{2} \mu \underline{\lambda}$. On the intervals where $m$ is continuous, the time derivative of $m$ is the solution of the following Cauchy problem

$$
\begin{aligned}
\dot{m}(t) & =-2 \alpha m(t)+q_{\sigma[w]\left(t_{k}\right)}(w(t)), \quad t \in\left[t_{k}, t_{t_{k+1}}\right), \\
m\left(t_{k}\right) & =0 .
\end{aligned}
$$

Thus the solution is

$$
m(t)=e^{-2 \alpha t} \int_{t_{k}}^{t} e^{2 \alpha s} q_{\sigma[w]\left(t_{k}\right)}(w(s)) d s, \quad t \in\left[t_{k}, t_{k+1}\right) .
$$

To sum up the control consists in keeping $m(t)$ negative or zero at any time. The motivation for the choice of the function $m$ comes from Gronwall's inequality [see for example [8, pages 708-709]].

The following lemma holds for the switching rule 67)- 71 .

Lemma 3. Under Assumption 1 with the strategy [67)-(71), if $w \in C_{r p w}\left(\mathbb{R}^{+}, \mathbb{R}^{n}\right)$ then $\sigma[w] \in C_{r p w}\left(\mathbb{R}^{+}, \mathcal{I}\right)$. 
Proof. Let $K$ be a compact subset of $\mathbb{R}^{+}$. Let us show that the number of discontinuities of $\sigma[w]$ is finite in $K$. By hypothesis $w$ has a finite number of discontinuities on $K$. Let $t_{1}, \ldots, t_{M} \in K$ be these times of discontinuity, and $t_{0}$ and $t_{M+1}$ are respectively the lower bound and the upper bound of the interval $K$.

The lines to prove the result are similar to those of the proof of Lemma 2 . In the same fashion we consider the continuation of $w$, instead of $w$ itself. But for the sake of simplicity we keep $w$ in our notation.

Pick $i \in\{0, \ldots, M\}$. Observe that on $\mathbb{R}^{n}, q_{k}$ is continuous, as $w$ on $\left[t_{i}, t_{i+1}\right]$, thus the functions $q_{k}(w)$ are continuous on the compact $\left[t_{i}, t_{i+1}\right]$ and therefore uniformly continuous. Using the fact that there is a finite number of functions $q_{k}(w)$ and the uniform continuity, there exists $\tau_{i}^{*}>0$ such that

$$
\forall k \in \mathcal{I}, \quad \forall \hat{t}_{i}, \check{t}_{i} \in\left[t_{i}, t_{i+1}\right]: \quad\left|\hat{t}_{i}-\check{t}_{i}\right| \leq \tau_{i}^{*} \Rightarrow\left|q_{k}\left(w\left(\hat{t}_{i}\right)\right)-q_{k}\left(w\left(\check{t}_{i}\right)\right)\right| \leq \varepsilon\left(t_{i+1}\right) .
$$

Without loss of generality we can choose $\tau_{i}^{*}$ such that $1-2 \alpha \leq e^{-2 \alpha \tau_{i}^{*}}$. Assume $\bar{t} \in\left[t_{i}, t_{i+1}\right]$ is a switching time, hence by Assumption 1 it holds $q_{\sigma[w](\bar{t})}(w(\bar{t})) \leq 0$, and one gets

$$
\forall \hat{t} \in\left[\bar{t}, t_{i+1}\right]: \quad|\bar{t}-\hat{t}| \leq \tau_{i}^{*} \Rightarrow\left|q_{\sigma[w](\bar{t})}(w(\bar{t}))-q_{\sigma[w](\bar{t})}(w(\hat{t}))\right| \leq \varepsilon\left(t_{i+1}\right) .
$$

The last inequality is equivalent to

$$
-\varepsilon\left(t_{i+1}\right)+q_{\sigma[w](\bar{t})}(w(\bar{t})) \leq q_{\sigma[w](\bar{t})}(w(\hat{t})) \leq \varepsilon\left(t_{i+1}\right)+q_{\sigma[w](\bar{t})}(w(\bar{t})) .
$$

Since $q_{\sigma[w](\bar{t})}(w(\bar{t})) \leq 0$ one gets

$$
q_{\sigma[w](\bar{t})}(w(\hat{t})) \leq \varepsilon\left(t_{i+1}\right) .
$$

With 77 one gets

$$
e^{-2 \alpha \hat{t}} \int_{\bar{t}}^{\hat{t}} e^{2 \alpha s} q_{\sigma[w](\bar{t})}(w(s)) d s \leq e^{-2 \alpha \hat{t}} \varepsilon\left(t_{i+1}\right) \int_{\bar{t}}^{\hat{t}} e^{2 \alpha s} d s, \quad \forall \hat{t} \in\left[\bar{t}, \bar{t}+\tau_{i}^{*}\right] .
$$

From $(78)$ it follows

$$
m(\hat{t}) \leq \frac{\left(1-e^{-2 \alpha(\hat{t}-\bar{t})}\right)}{2 \alpha} \varepsilon\left(t_{i+1}\right) \leq \varepsilon\left(t_{i+1}\right), \quad \forall \hat{t} \in\left[t_{i}, t_{i+1}\right] .
$$

Thus, the next switching time after $\bar{t}$ cannot appear before a time $\tau_{i}^{*}$. Then an upper bound for the maximal number of switches on $\left[t_{i}, t_{i+1}\right]$ is given by

$$
\bar{s}_{i}=\frac{t_{i+1}-t_{i}}{\tau_{i}^{*}}+1
$$

To conclude we get that the number of discontinuities of $\sigma[w]$ on $K$ is bounded by

$$
\bar{S}=\sum_{i=1}^{M} \bar{s}_{i}
$$

which is finite. The right continuity of $\sigma[w]$ follows from the strict inequality in the first line of $[69$. This concludes the proof of Lemma 3.

As in the last strategy we are able to give a result of global exponential convergence with the switching rule 67)-61).

Theorem 2. Under Assumption 1, system (1)-(2)-(3)-(9)-(10) with an initial condition $y^{0} \in C_{l p w}\left([0,1], \mathbb{R}^{n}\right)$ and the switching rule 67 - 71 is globally exponentially convergent with $\alpha=\frac{1}{2} \mu \underline{\lambda}$ and $g(r)=c(r+\sqrt{\varepsilon(0)})$ with $c>0$. 
Proof. The existence and uniqueness of a solution to the system (1)-(2)-(3)-(9)-(10) with the switching rule (67) - 71) is given by Lemma 3 and Proposition 1. Now to show that the system is globally convergent we have to etablish relation (26). Thanks to Lemma 1 the time derivative of $V$ along the solution of the system (1)-(2)-(3)-(9)-(10) is given by

$$
\dot{V} \leq-2 \alpha V+q_{\sigma[w](t)}(w(t)) .
$$

Thanks to differential form of Gronwall's Lemma one gets

$$
V \leq e^{-2 \alpha t} V\left(y^{0}\right)+e^{-2 \alpha t} \int_{0}^{t} e^{2 \alpha s} q_{\sigma[w](s)}(w(s)) d s .
$$

Using the linearity of the integral one gets

$$
V \leq e^{-2 \alpha t} V\left(y^{0}\right)+e^{-2 \alpha t}\left(\sum_{i=0}^{k-1} \int_{t_{i}}^{t_{i+1}} e^{2 \alpha s} q_{\sigma[w]\left(t_{i}\right)}(w(s)) d s+\int_{t_{k}}^{t} e^{2 \alpha s} q_{\sigma[w]\left(t_{k}\right)}(w(s)) d s\right)
$$

where the $t_{i}$ s are the switching times of $\sigma[w], t$ lies in a interswitching interval $\left(t_{k}, t_{k+1}\right)$. Let us point out that the number of them is finite thanks to Lemma 3 . Since $\varepsilon\left(t_{i+1}\right)=e^{-2 \alpha t_{i+1}} \int_{t_{i}}^{t_{i+1}} e^{2 \alpha s} q_{\sigma[w]\left(t_{i}\right)}(w(s)) d s$, one gets

$$
V \leq e^{-2 \alpha t} V\left(y^{0}\right)+e^{-2 \alpha t}\left(\sum_{i=0}^{k-1} e^{2 \alpha t_{i+1}} \varepsilon\left(t_{i+1}\right)+\int_{t_{k}}^{t} e^{2 \alpha s} q_{\sigma[w]\left(t_{k}\right)}(w(s)) d s\right) .
$$

Using the positivity and the continuity of $\varepsilon$, and the switching rule $67-71$ we have the following inequality for all $t \in\left(t_{k}, t_{k+1}\right)$

$$
V \leq e^{-2 \alpha t} V\left(y^{0}\right)+e^{-2 \alpha t}\left(\int_{0}^{t_{k}} e^{2 \alpha s} \varepsilon(s) d s+e^{2 \alpha t} \varepsilon(t)\right) .
$$

Using the positivity of $\varepsilon$ one gets

$$
\begin{aligned}
& V \leq e^{-2 \alpha t} V\left(y^{0}\right)+e^{-2 \alpha t}\left(\int_{0}^{t_{k}} e^{2 \alpha s} \varepsilon(s) d s+\int_{t_{k}}^{t} e^{2 \alpha s} \varepsilon(s) d s+e^{2 \alpha t} \varepsilon(t)\right), \\
& V \leq e^{-2 \alpha t} V\left(y^{0}\right)+\left(1+\frac{1}{2 \alpha-\eta}\right) \varepsilon(0) e^{-\eta t}-\frac{\varepsilon(0)}{2 \alpha-\eta} e^{-2 \alpha t} .
\end{aligned}
$$

Letting $c=\max \left\{\sqrt{\frac{\kappa}{\nu \delta}}, \sqrt{\frac{\eta-2 \alpha+1}{\nu \delta(\eta-2 \alpha)}}\right\}$ one gets 26 with $g(r)=c(r+\sqrt{\varepsilon(0)})$, and thus the global exponential convergence of (1)-(2)-(3)-(9)-(10) with the switching rule $(69)$ follows.

This concludes the proof of Theorem 2

\section{Robustness with respect to measurement noise}

Motivated by the idea to insure the stability with respect to measurement noise, we consider system (1)-(2)(3) - (9)-(47) with the switching rule

$$
\begin{aligned}
m(0) & =0, \\
\lim _{\tau \rightarrow t^{+}} m(\tau) & = \begin{cases}m(t) & \text { if } m(t)<-\zeta e^{-2 \alpha t} \int_{t_{k}}^{t} e^{2 \alpha s}|w(s)+\delta(s)|^{2} d s+\varepsilon(t), \\
0 & m(t)=-\zeta e^{-2 \alpha t} \int_{t_{k}}^{t} e^{2 \alpha s}|w(s)+\delta(s)|^{2} d s+\varepsilon(t),\end{cases} \\
\sigma[w+\delta](t) & = \begin{cases}\sigma[w+\delta]\left(t^{-}\right) & \text {if } m(t)<-\zeta e^{-2 \alpha t} \int_{t_{k}}^{t} e^{2 \alpha s}|w(s)+\delta(s)|^{2} d s+\varepsilon(t), \\
\underset{j \in\{1, \ldots, N\}}{\arg \min } q_{j}(w(t)+\delta(t)) & \text { if } m(t)=-\zeta e^{-2 \alpha t} \int_{t_{k}}^{t} e^{2 \alpha s}|w(s)+\delta(s)|^{2} d s+\varepsilon(t),\end{cases} \\
\sigma[w+\delta](0) & =\underset{j \in\{1, \ldots, N\}}{\arg \min q_{j}(w(0)+\delta(0)),} \\
\dot{\varepsilon}(t) & =-\eta \varepsilon(t), \quad \varepsilon(0)>0,,
\end{aligned}
$$


where $t_{k}$ is the first instant before $t$ for which $m$ vanishes and $\eta$ is such that $\eta>2 \alpha, \alpha=\frac{1}{2} \mu \underline{\lambda}$. On the intervals where $m$ is continuous, the time derivative of $m$ is the solution of the following Cauchy problem

$$
\begin{aligned}
\dot{m}(t) & =-2 \alpha m(t)+q_{\sigma[w+\delta]\left(t_{k}\right)}(w(t)+\delta(t)), t \in\left[t_{k}, t_{k+1}\right), \\
m\left(t_{k}\right) & =0 .
\end{aligned}
$$

Thus the solution is

$$
m(t)=e^{-2 \alpha t} \int_{t_{k}}^{t} e^{2 \alpha s} q_{\sigma[w+\delta](s)}(w(s)+\delta(s)) d s, \quad t \in\left[t_{k}, t_{k+1}\right) .
$$

Let us state the following result.

Proposition 4. Under Assumption 2, system (1)-(2)-(3)-(9)-(47) with an initial condition $y^{0} \in C_{l p w}\left([0,1], \mathbb{R}^{n}\right)$ and a disturbance $\delta \in C_{r p w}\left(\mathbb{R}^{+}, \mathbb{R}^{n}\right)$ satisfying

$$
|\delta(t)| \leq \rho|w(t)|, \quad \forall t \in \mathbb{R}^{+},
$$

for a positive constant $\rho$ such that

$$
0<\rho \leq \sqrt{1+\frac{\zeta}{\beta}}-1
$$

with the switching rule 85-89) is globally exponentially convergent.

Proof. First note that Lemma 3 is still valid in the context of the switching rule $85-(89)$ instead of (67)-(71), for any $\delta \in C_{r p w}\left(\mathbb{R}^{+}, \mathbb{R}^{n}\right)$. Hence the existence and uniqueness of a solution to the system (1)-(2)-(3)-(9)(47) with the switching rule $85-89$ is given by Lemma 3 and Proposition 1. Due to Lemma 1 the time derivative of $V$ is given by

$$
\dot{V} \leq-2 \alpha V+q_{\sigma[w+\delta](t)}(w(t)) .
$$

Thank to differential form of Gronwall's Lemma one gets

$$
V \leq e^{-2 \alpha t} V\left(y^{0}\right)+e^{-2 \alpha t} \int_{0}^{t} e^{2 \alpha s} q_{\sigma[w+\delta](s)}(w(s)) d s .
$$

Using (58), relation (96) becomes

$$
\begin{aligned}
V \leq & e^{-2 \alpha t} V\left(y^{0}\right)+e^{-2 \alpha t} \int_{0}^{t} e^{2 \alpha s} q_{\sigma[w+\delta](s)}(w(s)+\delta(s)) d s \\
& -e^{-2 \alpha t} \int_{0}^{t} e^{2 \alpha s} \delta(s)^{\top}\left(G_{\sigma[w+\delta](s)}^{\top} Q \Lambda(t) G_{\sigma[w+\delta](s)}-e^{-\mu} Q \Lambda(t)\right)(2 w(s)+\delta(s)) d s .
\end{aligned}
$$

Let us denote the second term in the right-hand side of (97) by $R(t)$. Using the linearity of the integral one gets

$$
R(t)=e^{-2 \alpha t}\left(\sum_{i=0}^{k-1} \int_{t_{i}}^{t_{i+1}} e^{2 \alpha s} q_{\sigma[w+\delta](s)}(w(s)+\delta(s)) d s+\int_{t_{k}}^{t} e^{2 \alpha s} q_{\sigma[w+\delta](s)}(w(s)+\delta(s)) d s\right) d s .
$$

where the $t_{i}$ s are the switching times of $\sigma[w+\delta], t$ lies in a interswitching interval $\left(t_{k}, t_{k+1}\right)$. Let us point out that the number of them is finite thank to Lemma 3 . Since $m\left(t_{i+1}\right)=-\zeta e^{-2 \alpha t_{i+1}} \int_{t_{i}}^{t_{i+1}} e^{2 \alpha s}|w(s)+\delta(s)|^{2} d s+$ $\varepsilon\left(t_{i+1}\right), 98$ becomes

$$
\begin{aligned}
R(t)= & e^{-2 \alpha t}\left(\sum_{i=0}^{k-1}-\zeta \int_{t_{i}}^{t_{i+1}} e^{2 \alpha s}|w(s)+\delta(s)|^{2} d s+e^{2 \alpha t_{i+1}} \varepsilon\left(t_{i+1}\right)+\int_{t_{k}}^{t} e^{2 \alpha s} q_{\sigma[w+\delta](s)}(w(s)+\delta(s)) d s\right) \\
= & -\zeta e^{-2 \alpha t} \int_{0}^{t_{k}} e^{2 \alpha s}|w(s)+\delta(s)|^{2} d s \\
& +e^{-2 \alpha t}\left(\sum_{i=0}^{k-1} e^{2 \alpha t_{i+1}} \varepsilon\left(t_{i+1}\right)+\int_{t_{k}}^{t} e^{2 \alpha s} q_{\sigma[w+\delta](s)}(w(s)+\delta(s)) d s\right)
\end{aligned}
$$


Using the positivity and the continuity of $\varepsilon$, and the switching rule $85-89$ we have the following inequality for all $t \in\left(t_{k}, t_{k+1}\right)$

$$
R(t) \leq-\zeta e^{-2 \alpha t} \int_{0}^{t} e^{2 \alpha s}|w(s)+\delta(s)|^{2} d s+e^{-2 \alpha t} \int_{0}^{t} e^{2 \alpha s} \varepsilon(s) d s
$$

Hence

$$
\begin{aligned}
V \leq & e^{-2 \alpha t} V\left(y^{0}\right)-\zeta e^{-2 \alpha t} \int_{0}^{t} e^{2 \alpha s}|w(s)+\delta(s)|^{2} d s+e^{-2 \alpha t} \int_{0}^{t} e^{2 \alpha s} \varepsilon(s) d s \\
& -e^{-2 \alpha t} \int_{0}^{t} e^{2 \alpha s} \delta(s)^{\top}\left(G_{\sigma[w+\delta](s)}^{\top} Q \Lambda(t) G_{\sigma[w+\delta](s)}-e^{-\mu} Q \Lambda(t)\right)(2 w(s)+\delta(s)) d s .
\end{aligned}
$$

By the proof of Proposition 3 we know that

$$
\begin{aligned}
& -e^{-2 \alpha t} \int_{0}^{t} e^{2 \alpha s} \delta(s)^{\top}\left(G_{\sigma[w+\delta](s)}^{\top} Q \Lambda(t) G_{\sigma[w+\delta](s)}-e^{-\mu} Q \Lambda(t)\right)(2 w(s)+\delta(s)) d s \\
& -\zeta e^{-2 \alpha t} \int_{0}^{t} e^{2 \alpha s}|w(s)+\delta(s)|^{2} d s \leq\left(\beta \rho^{2}+2 \beta \rho-\zeta\right) e^{-2 \alpha t} \int_{0}^{t} e^{2 \alpha s}|w(s)|^{2} d s .
\end{aligned}
$$

Analogously to the proof of Proposition 3 the polynomial $\beta \rho^{2}+2 \beta \rho-\zeta$ in $\rho$ has a positive root given by

$$
\rho=\sqrt{1+\frac{\zeta}{\beta}}-1
$$

Hence for all choice of $\rho$ such that 94 holds we get

$$
V \leq e^{-2 \alpha t} V\left(y^{0}\right)+e^{-2 \alpha t} \int_{0}^{t} e^{2 \alpha s} \varepsilon(s) d s .
$$

The end of the proof follows the proof of Theorem 2. This concludes the proof of Proposition 4 .

\section{Examples}

\subsection{Abstract example}

The merits of the three switching rules have been illustrated in [17, though the velocities were constant and $\varepsilon(t) \equiv 0$. To illustrate Proposition 3 and 4 , let us consider system $(1)-(2)-(3)$ with two modes $(\mathcal{I}=\{1,2\})$. The initial conditions are selected as the first three elements of an orthonormal basis of $L^{2}\left([0,1], \mathbb{R}^{2}\right)$. More specifically the following three initial conditions

$$
y_{k}^{0}(x)=\left(\begin{array}{c}
\sqrt{2} \sin ((2 k-1) \pi x) \\
\sqrt{2} \sin (2 k \pi x)
\end{array}\right), k=1,2,3,
$$

are considered. The matrix of the system (1) is given by

$$
\Lambda(t)=\operatorname{diag}(0.5+0.05 \sin (10 t), 0.5+0.05 \cos (10 t)), \quad t \in \mathbb{R}^{+} .
$$

Thanks to a result of [4, boundary matrices $G_{i}$ which destabilize the unswitched system must satisfy $\rho\left(\left|G_{i}\right|\right)>1^{1}$. The instability of the system with the proposed matrices is checked numerically with a Weighted Essentially Non Oscillatory scheme (see [19]). Boundary matrices $G_{1}$ and $G_{2}$ are proposed as

$$
G_{1}=\left(\begin{array}{cc}
1.1 & 0 \\
-0.3 & 0.1
\end{array}\right), \quad G_{2}=\left(\begin{array}{cc}
0 & 0.2 \\
0.1 & -1.05
\end{array}\right)
$$

\footnotetext{
${ }^{1}$ For the time-invariant velocity case that is $\Lambda(t) \equiv \Lambda$, one has: if $\rho(|G|)<1$ then the system $\sqrt{1}$, $\sqrt{2}$ is exponentially stable (see for instance [4). Since the velocity perturbation is "small" it is natural to search matrices $G$ which do not satisfy the previous condition in order to destabilize the system. For more discussions about conditions on $G$ see [4].
} 
The respective spectrum of $\left|G_{1}\right|$ and $\left|G_{2}\right|$ is $\{0.1 ; 1.1\}$ and $\{-0.0187 ; 1.0687\}$.

To illustrate our result of robustness let us set $\zeta=0.07, \mu=0.01, Q=\operatorname{diag}(1.1231,1)$. With $\gamma_{1}=0.5$ (hence $\gamma_{2}=0.5$ ) it is obtained

$$
\sum_{i=1}^{2} \gamma_{i}\left(G_{i}^{\top} Q \bar{\Lambda} G_{i}-e^{-\mu} Q \underline{\Lambda}+\zeta I_{2}\right)=\left(\begin{array}{cc}
-0.0292 & -0.0371 \\
-0.0371 & -0.0572
\end{array}\right)
$$

The eigenvalues of the previous matrix are -0.0829 and -0.0035 . Therefore it is a symmetric negative definite matrix, and Assumption 2 is satisfied. The computation of $\beta$ gives 0.5405 . One set the error margin as the maximum margin allowed by Proposition 3 , that is $\rho=6.28 \%$.

See Figure 2 for the time evolution of the solution with constant control input $\bar{i}=1$ and the initial condition $y_{2}^{0}$ where the instability is observed.

The function $\varepsilon$ used in the switching rules $(50)-(52)$ and $\sqrt{85}-(89)$ is chosen to be

$$
\varepsilon(t)=10^{-3} e^{-t}, \quad t \in \mathbb{R}^{+} .
$$

The measurement noise is chosen as follows

$$
\delta(t)=\|w(t)\| \rho(\cos (t), \sin (t))^{\top} .
$$

As it was expected the solution is stabilized when switching rules $(50)-(52)$ and $(85)-89)$ are applied to the system under measurement noise. Figure 3 shows the time evolution of the two components of the solution with the switching rules (50). Numerically the rate of convergence $\alpha$ is equal to 0.4327 .
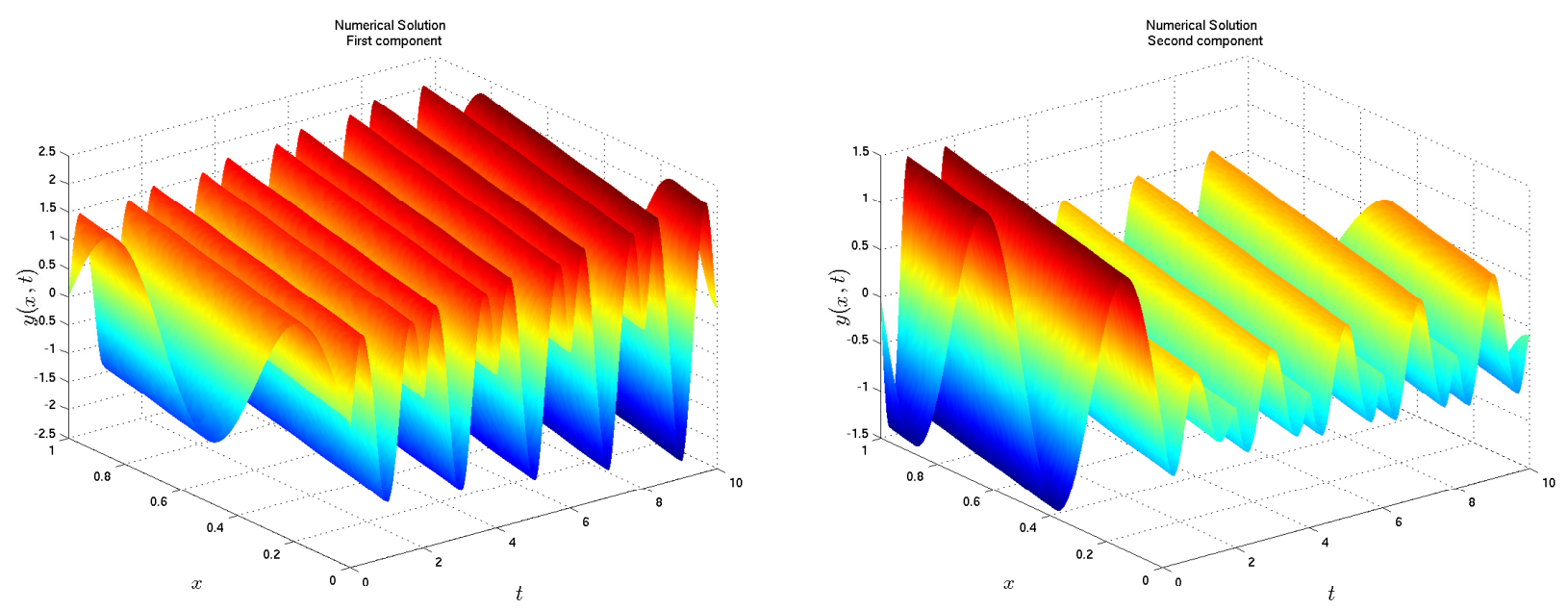

Figure 2: Time evolution of the first component $y_{1}$ (left) and of the second component $y_{2}$ (right) of the solution of the unswitched system (1)-22)-(3) with the active mode $\bar{i}=1$. 

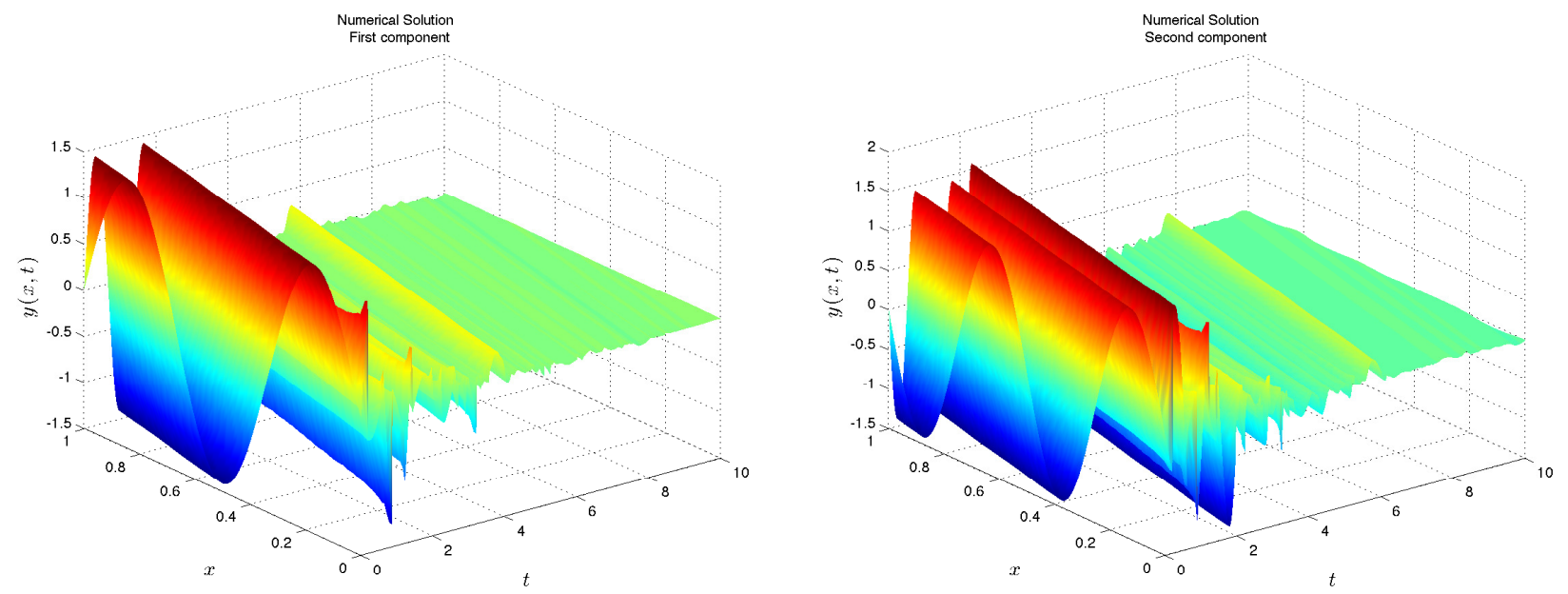

Figure 3: Time evolution of the first component $y_{1}$ (left) and of the second component $y_{2}$ (right) of the solution of (1)-(2)- 3 - $-(9)-(10)$ with the switching rule $\sqrt{50}-(52)$ with measurement noise $(110)$.

For a discussion on the impact of the $\mu$ parameter on the rate of convergence, and the number of switches per time unit, see [17].

\subsection{Application: switched boundary controllers for the Saint-Venant equations}

\subsubsection{Saint-Venant equations for a network of open channels}

The previous illustration was motivated by theoretical objectives. In this section, we introduce a more physical system. Let us consider a cascade of $\mathcal{M}$ canal reaches. Each reach is a one-dimensional pool with a rectangular cross-section, a unit width and a zero slope. Moreover we suppose that each reach has the same length $L$. Besides, the friction effects due to the walls are neglected. The dynamics of the system in each reach is then given by the so-called Saint-Venant equations

$$
\partial_{t}\left(\begin{array}{c}
H_{i} \\
V_{i}
\end{array}\right)+\partial_{x}\left(\begin{array}{c}
H_{i} V_{i} \\
\frac{V_{i}^{2}}{2}+g H_{i}
\end{array}\right)=0, \quad x \in(0, L), \quad i=1, \ldots, \mathcal{M},
$$

where $H_{i}$ and $V_{i}$ denoted respectively the water depth and the velocity in the reach $i$. In those equations $g$ is the gravity constant. Moreover the flow-rate in these pools can be defined by

$$
Q_{i}(t, x)=H_{i}(t, x) V_{i}(t, x), \quad i=1, \ldots, \mathcal{M} .
$$

In order to have a linear hyperbolic system in the form (1)-(2)-(3), we linearized the system around a steady-state and made the system dimensionless in the space variable. A steady-state solution is a constant solution

$$
H_{i}(t, x)=H_{i}^{*}, \quad V_{i}(t, x)=V_{i}^{*}, \quad i=1, \ldots, \mathcal{M}, \quad \forall t \in \mathbb{R}^{+}, \forall x \in[0, L] .
$$

The steady-state flow is assumed to be subcritical or fluvial that is

$$
g H_{i}^{*}-V_{i}^{*^{2}}>0 \text {. }
$$

\subsubsection{Linearization}

We define the deviations of the state $H_{i}(t, x), V_{i}(t, x), i=1, \ldots, \mathcal{M}$, w.r.t. the steady-state as

$$
\begin{aligned}
h_{i}(t, x) & =H_{i}(t, x)-H_{i}^{*}, \\
v_{i}(t, x) & =V_{i}(t, x)-V_{i}^{*} .
\end{aligned}
$$


Let us turn to dimensionless form. We define the new variable

$$
\tilde{x}=\frac{x}{L} \text {. }
$$

Then, for each reach $i$, the system 111 linearized around the steady-state, is expressed with the new space variable $\tilde{x}$ as

$$
\partial_{t} \tilde{\zeta}_{i}+\tilde{A}_{i}\left(\tilde{\zeta}_{i}^{*}\right) \partial_{\tilde{x}} \tilde{\zeta}_{i}=0, \quad i=1, \ldots, \mathcal{M}
$$

where

$$
\tilde{\zeta}_{i}=\left(\begin{array}{c}
\tilde{h}_{i} \\
\tilde{v}_{i}
\end{array}\right), \quad \tilde{A}_{i}\left(\tilde{\zeta_{i}^{*}}\right)=\left(\begin{array}{cc}
\frac{V_{i}^{*}}{L} & \frac{H_{i}^{*}}{L} \\
\frac{g}{L} & \frac{V_{i}^{*}}{L}
\end{array}\right), \quad \tilde{h}_{i}(t, \tilde{x})=h_{i}(t, L \tilde{x}), \quad \tilde{v}_{i}(t, \tilde{x})=v_{i}(t, L \tilde{x})
$$

The eigenvalues $\lambda_{i}$ and $\lambda_{i+\mathcal{M}}, i=1, \ldots, \mathcal{M}$, of $\tilde{A}_{i}\left(\tilde{\zeta}_{i}^{*}\right)$ are

$$
\lambda_{i}=\frac{1}{L}\left(V_{i}^{*}+\sqrt{g H_{i}^{*}}\right), \quad \lambda_{i+\mathcal{M}}=\frac{1}{L}\left(V_{i}^{*}-\sqrt{g H_{i}^{*}}\right) .
$$

Under the condition 113 the system is strictly hyperbolic with

$$
\lambda_{i+\mathcal{M}}<0<\lambda_{i}, \quad i=1, \ldots, \mathcal{M}
$$

The corresponding left eigenvectors of $\tilde{A}_{i}$ are

$$
l_{i}=\left(\sqrt{\frac{g}{H_{i}^{*}}}, 1\right), \quad l_{i+\mathcal{M}}=\left(-\sqrt{\frac{g}{H_{i}^{*}}}, 1\right) .
$$

Multiplying (117) by $l_{i}$ and $l_{i+\mathcal{M}}$, the subsystems (117) are rewritten in the characteristic coordinates

$$
\partial_{t} \mathbf{y}_{i}+\hat{A}_{i}\left(\mathbf{y}_{i}^{*}\right) \partial_{x} \mathbf{y}_{i}=0
$$

where $\mathbf{y}_{i}=\left(y_{i}, y_{i+\mathcal{M}}\right)^{\top}$ is such that

$$
\begin{aligned}
y_{i} & =\sqrt{\frac{g}{H_{i}^{*}}} \tilde{h}_{i}+\tilde{v}_{i}, \\
y_{i+\mathcal{M}} & =-\sqrt{\frac{g}{H_{i}^{*}}} \tilde{h}_{i}+\tilde{v}_{i},
\end{aligned}
$$

and $\hat{A}_{i}\left(\mathbf{y}_{i}^{*}\right)=\operatorname{diag}\left(\lambda_{i}, \lambda_{i+\mathcal{M}}\right)$. Conversely $\tilde{v}_{i}$ and $\tilde{h}_{i}$ can be expressed in function of $y_{i}$ and $y_{i+\mathcal{M}}$

$$
\begin{aligned}
\tilde{h}_{i} & =\frac{1}{2} \sqrt{\frac{H_{i}^{*}}{g}}\left(y_{i}-y_{i+\mathcal{M}}\right), \\
\tilde{v}_{i} & =\frac{1}{2}\left(y_{i}+y_{i+\mathcal{M}}\right) .
\end{aligned}
$$

We introduce the vectors $y^{+}=\left(y_{1}, \ldots, y_{\mathcal{M}}\right)^{\top}$ and $y^{-}=\left(y_{1+\mathcal{M}}, \ldots, y_{2 \mathcal{M}}\right)^{\top}$, and we denoted $y=\left(y^{+}, y^{-}\right)^{\top}$ the solution to the whole system

$$
\partial_{t} y+\Lambda \partial_{x} y=0
$$

where $\Lambda=\operatorname{diag}\left(\lambda_{1}, \ldots, \lambda_{2 \mathcal{M}}\right)$. 


\subsubsection{Boundary conditions}

System (123) is subject to a set of boundary conditions, as in 2. First of all there are $\mathcal{M}-1$ conditions which express the flow conservation between the pools

$$
H_{i}(t, 1) V_{i}(t, 1)=H_{i+1}(t, 0) V_{i+1}(t, 0), \quad i=1, \ldots, \mathcal{M}-1 .
$$

Then one adds a condition which imposes the inflow rate

$$
H_{1}(t, 0) V_{1}(t, 0)=Q_{0}(t) .
$$

In the next we impose a constant inflow rate, that is $Q_{0}(t)=Q^{*}=H_{1}^{*} V_{1}^{*}$. The last $\mathcal{M}$ boundary conditions are given by the gate operations. We are considering underflow sluice gates with corresponding gate openings $u_{i}^{j}$ for reach $i \in\{1, \ldots, \mathcal{M}\}$ in mode $j \in \mathcal{I}$. The discharge relationship is given by

$$
H_{i}(t, 1) V_{i}(t, 1)=u_{i}^{j} \mu_{0} l \sqrt{2 g\left(H_{i}(t, 1)-H_{i+1}(t, 0)\right)}, \quad i=1, \ldots, \mathcal{M}-1,
$$

where $\mu_{0}$ is a positive constant coefficient and $l$ is the channel width (here $l=1 \mathrm{~m}$ ). For the last gate we have

$$
H_{\mathcal{M}}(t, 1) V_{\mathcal{M}}(t, 1)=u_{\mathcal{M}}^{j} \mu_{0} l \sqrt{2 g\left(H_{\mathcal{M}}(t, 1)-H_{\text {down }}\right)},
$$

where $H_{\text {down }}>0$ denotes the constant downstream water level. In the Riemann coordinates, boundary conditions (126) and 127) are equivalent to

$$
y_{i+\mathcal{M}}(t, 1)=-k_{i}^{j} y_{i}(t, 1), \quad i=1, \ldots, \mathcal{M}, j=1, \ldots, N,
$$

for a suitable choice of the control actions $u_{i}^{j}(t)$,

$$
\begin{aligned}
u_{i}^{j}(t) & =\frac{H_{i}(t, 1)\left(\left(\frac{1-k_{i}^{j}}{1+k_{i}^{j}}\right) \sqrt{\frac{g}{H_{i}^{*}}}\left(H_{i}(t, 1)-H_{i}^{*}\right)+V_{i}^{*}\right)}{\mu_{0} l \sqrt{2 g\left(H_{i}(t, 1)-H_{i+1}(t, 0)\right)}}, \quad i=1, \ldots, \mathcal{M}-1, \\
u_{\mathcal{M}}^{j}(t) & =\frac{H_{\mathcal{M}}(t, 1)\left(\left(\frac{1-k_{\mathcal{M}}^{j}}{1+k_{\mathcal{M}}^{j}}\right) \sqrt{\frac{g}{H_{\mathcal{M}}^{*}}}\left(H_{\mathcal{M}}(t, 1)-H_{\mathcal{M}}^{*}\right)+V_{\mathcal{M}}^{*}\right)}{\mu_{0} l \sqrt{2 g\left(H_{\mathcal{M}}(t, 1)-H_{\text {down }}\right)}} .
\end{aligned}
$$

The values $k_{i}^{j}$ are tuning parameters. Obviously the control actions are well defined only if $H_{i}(t, 1)>$ $H_{i+1}(t, 0)$ for all $i=1, \ldots, \mathcal{M}-1$ and $H_{\mathcal{M}}(t, 1)>H_{\text {down }}$. The linearization of the boundary conditions provided $N$ matrices such that

$$
\left(\begin{array}{c}
y^{+}(t, 0) \\
y^{-}(t, 1)
\end{array}\right)=\left(\begin{array}{cc}
G_{j}^{++} & G_{j}^{+-} \\
G_{j}^{-+} & G_{j}^{--}
\end{array}\right)\left(\begin{array}{c}
y^{+}(t, 1) \\
y^{-}(t, 0)
\end{array}\right), \quad t \in\left[t_{j}, t_{j+1}\right]
$$

Denoted $G_{j}=\left(\begin{array}{cc}G_{j}^{++} & G_{j}^{+-} \\ G_{j}^{-+} & G_{j}^{--}\end{array}\right)$. The matrices $G_{j}^{-+}$and $G_{j}^{--}$are given by conditions 128 as

$$
G_{j}^{-+}=\operatorname{diag}\left\{-k_{i}, i=1, \ldots, \mathcal{M}\right\}, \quad G_{j}^{--}=0, \quad j=1, \ldots, N .
$$

The conditions 124 and 125 give the following expression for the matrices $G_{j}^{+-}$

$$
G_{j}^{+-}=\operatorname{diag}\left\{\frac{\lambda_{i+\mathcal{M}}}{\lambda_{i}}, i=1, \ldots, \mathcal{M}\right\}, \quad j=1, \ldots, N .
$$

Finally, the matrices $G_{j}^{++}$are obtained thanks to the conditions 124 and 128 as

$$
G_{j}^{++}[i+1, i]=\frac{\left(\lambda_{i}+k_{i}^{j} \lambda_{i+\mathcal{M}}\right)}{\lambda_{i+1}} \sqrt{\frac{H_{i}^{*}}{H_{i+1}^{*}}} \text { and } 0 \text { elsewhere, } \quad i=1, \ldots, \mathcal{M} .
$$




\subsubsection{Simulation experiments}

The switched strategies developped in this paper are now tested with some numerical simulations in the case presented above. To this end, we consider two pools whose parameters are $L=1000 \mathrm{~m}$, width $l=1$ $\mathrm{m}, H_{\text {down }}=0.2 \mathrm{~m}, \mu_{0}=0.4,\left(H_{1}^{*}, H_{2}^{*}\right)=(2.5,1) \mathrm{m}$ and $Q^{*}=1 \mathrm{~m}^{3} \cdot \mathrm{s}^{-1}$. The initial conditions are, for $x \in[0, L],\left(H_{1}(0, x), H_{2}(0, x)\right)=(4,1.4) \mathrm{m}, Q(0, x)=2 \mathrm{~m}^{3} \cdot \mathrm{s}^{-1}$. There are two modes. The control gains associated to each mode are $\left(k_{1}^{1}, k_{2}^{1}\right)=(1,0.2)$ and $\left(k_{1}^{2}, k_{2}^{2}\right)=(0.2,1)$. The velocities are constant.

Assumption 1 is satisfied with $\mu=0.775, Q=10^{3} \mathrm{diag}(1.448,0.001,2.674,0.001)$ and $\gamma_{1}=0.95$.

The Saint-Venant equations are integrated numerically using the same scheme as in the Section 4.1 . In Figure 4 the evolution of the function $V$ is represented for the argmin and the argmin with hysteresis switching rules, with the constant mode $\sigma(t)=1$ and with the constant mode $\sigma(t)=2$. In this figure, we can observe that the switching strategies stabilize the system, and more importantly seem to improve the convergence of the system to the desired steady-state. Moreover, the switching signal for the argmin with hysteresis switching rule is displayed. It can be observed that the system keeps the mode 1 during the first $1200 \mathrm{~s}$, and then start to switch. Numerically, we observe that this behavior corresponds to the choice of stabilizing the first pool then the second one. Indeed the mode $i=1$ is the most efficient to stabilize the first pool. The argmin with hysteresis and filter keeps the mode 2 .

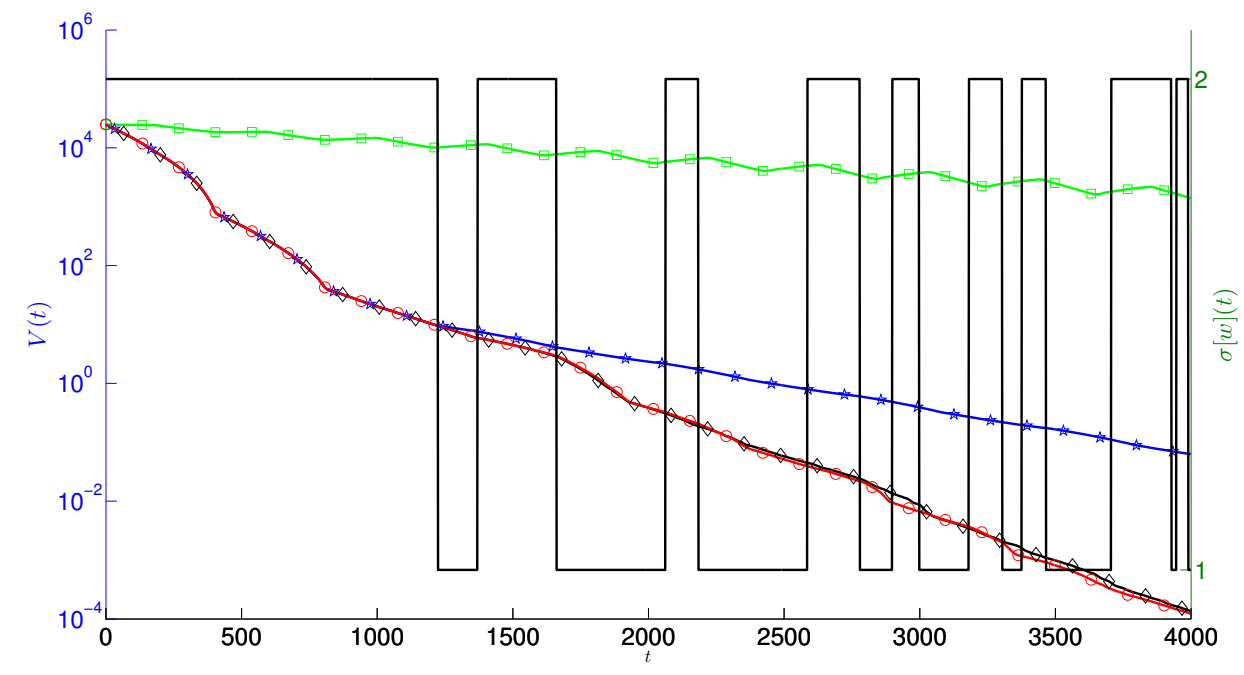

Figure 4: Evolution of the function $V$ (semilog scale) and of $\sigma[w]$ for the argmin with hysteresis switching rule. Legend: square marker for constant mode $\sigma(t)=1$, star marker for constant mode $\sigma(t)=2$, circle marker for the argmin with hysteresis switching rule and diamond marker for the argmin switching rule.

\section{Conclusion}

In this paper, three switching rules to stabilize hyperbolic systems of conservation laws of the form (1)-(2)(3) have been derived. These switching rules correspond to an output feedback law. The analysis of global exponential convergence is based on Lyapunov technique. This method has been adapted to the design problem of a stabilizing switching rule. The three switched controllers gave different performances which have been discussed and compared by some numerical simulations, especially with a network of open-channels governed by the Saint-Venant equations.

The first switching rule is based on an argmin computation. It allows to maximize the decay rate of the Lyapunov function at any time. In order to decrease the number of switches in a time interval, a hysteresis strategy to this rule was added. It means that the system stays in the current mode, as long as the Lyapunov function decreases. In order to reduce again the number of switches a low-pass filter was added to the former rule. We have proved the well-posedness with the argmin with hysteresis strategy and with the argmin, 
hysteresis and filter strategy. A sufficient condition on the matrices $G_{i}, \bar{\Lambda}$ and $\underline{\Lambda}$ has been derived to get the global exponential convergence of the system with these three switching rules. With mild modifications on the former sufficient condition, and on the argmin with hysteresis and argmin, hysteresis and filter, it has been proved that the system is exponentially convergent in presence of measurement noise with these switching rules.

\section{References}

[1] S. Amin, F. M. Hante, and A. M. Bayen. Exponential stability of switched linear hyperbolic initialboundary value problems. IEEE Trans. Automat. Contr., 57(2):291-301, 2012.

[2] G. Bastin, J.-M. Coron, and B. d'Andréa Novel. On Lyapunov stability of linearised Saint-Venant equations for a sloping channel. Netw. Heterog. Media, 4(2):177-187, 2009.

[3] J.-M. Coron. Control and Nonlinearity. Mathematical surveys and monographs. American Mathematical Society, 2009.

[4] J.-M. Coron, G. Bastin, and B. d'Andréa Novel. Dissipative boundary conditions for one-dimensional nonlinear hyperbolic systems. SIAM J. Control Optim., 47(3):1460-1498, 2008.

[5] J. de Halleux, C. Prieur, J.-M. Coron, B. d'Andréa Novel, and G. Bastin. Boundary feedback control in networks of open channels. Automatica, 39(8):1365-1376, 2003.

[6] A. Diagne, G. Bastin, and J.-M. Coron. Lyapunov exponential stability of 1-d linear hyperbolic systems of balance laws. Automatica, 48:109-114, 2012.

[7] V. Dos Santos and C. Prieur. Boundary control of open channels with numerical and experimental validations. IEEE Trans. on Control Systems Technology, 16(6):1252-1264, 2008.

[8] L.C. Evans. Partial Differential Equations. Graduate Studies in Mathematics. American Mathematical Society, 2010.

[9] J. C. Geromel and P. Colaneri. Stability and stabilization of discrete time switched systems. International Journal of Control, 79(7):719-728, 2006.

[10] M. Gugat, M. Dick, and G. Leugering. Gas flow in fan-shaped networks: Classical solutions and feedback stabilization. SIAM Journal on Control and Optimization, 49(5):2101-2117, 2011.

[11] M. Gugat and M. Sigalotti. Stars of vibrating strings: switching boundary feedback stabilization. Networks and Heterogeneous Media, 5(2):299-314, 2010.

[12] F. M. Hante, G. Leugering, and T. I. Seidman. Modeling and analysis of modal switching in networked transport systems. Appl. Math. Optim., 59(2):275-292, 2009.

[13] F. M. Hante, G. Leugering, and T. I. Seidman. An augmented BV setting for feedback switching control. Journal of Systems Science and Complexity, 23(3):456-466, 2010.

[14] F. M. Hante and M. Sigalotti. Converse Lyapunov theorems for switched systems in Banach and Hilbert spaces. SIAM J. Control Optim., 49(2):752-770, 2011.

[15] B. Haut and G. Bastin. A second order model of road junctions in fluid models of traffic networks. Networks and Heterogeneous Media, 2(2):227-257, 2007.

[16] M. Herceg, M. Kvasnica, C.N. Jones, and M. Morari. Multi-Parametric Toolbox 3.0. In Proc. of the European Control Conference, pages 502-510, Zürich, Switzerland, 2013. http://control.ee.ethz. $\mathrm{ch} / \sim \mathrm{mpt}$. 
[17] P.-O. Lamare, A. Girard, and C. Prieur. Lyapunov techniques for stabilization of switched linear systems of conservation laws. In Decision and Control (CDC), 2013 IEEE 52nd Annual Conference on, pages 448-453, Florence, Italy, 2013.

[18] D. Liberzon. Switching in systems and control. Springer, 2003.

[19] Xu-Dong Liu, Stanley Osher, and Tony Chan. Weighted essentially non-oscillatory schemes. Journal of computational physics, 115(1):200-212, 1994.

[20] Stefan Pettersson and Bengt Lennartson. Controller design of hybrid systems. In Hybrid and Real-Time Systems, pages 240-254. Springer, 1997.

[21] C. Prieur. A robust globally asymptotically stabilizing feedback: the example of the Artstein's circles. In Nonlinear control in the Year 2000, volume 258, pages 279-300. Springer Verlag London, 2001.

[22] C. Prieur. Asymptotic controllability and robust asymptotic stabilizability. SIAM Journal on Control and Optimization, 43(5):1888-1912, 2005.

[23] C. Prieur, A. Girard, and E. Witrant. Stability of switched linear hyperbolic systems by Lyapunov techniques. IEEE Transactions on Automatic Control, 59(8):2196-2202, 2014.

[24] C. Prieur and E. Trélat. Quasi-optimal robust stabilization of control systems. SIAM Journal on Control and Optimization, 45(5):1875-1897, 2006.

[25] T. I. Seidman. Feedback modal control of partial differential equations. In Optimal Control of Coupled Systems of Partial Differential Equations, volume 158 of International Series of Numerical Mathematics, pages 239-253. Birkhäuser Basel, 2009.

[26] E. D. Sontag and Y. Wang. On characterizations of the input-to-state stability property. Systems 8 Control Letters, 24(5):351-359, 1995.

[27] Jeremy G. VanAntwerp and Richard D. Braatz. A tutorial on linear and bilinear matrix inequalities. Journal of Process Control, 10(4):363-385, 2000. 